\title{
Influence of Age, Sex, and Diet on the human fecal metabolome investigated by ${ }^{1} \mathrm{H}$ NMR spectroscopy
}

Mengni Cui ${ }^{1}$, Alessia Trimigno ${ }^{1}$, Violetta Aru ${ }^{1}$, Morten A. Rasmussen ${ }^{1,2}$, Bekzod Khakimov ${ }^{1,}$ *, Søren Balling Engelsen 1, *

${ }^{1}$ Chemometrics and Analytical Technology Section Department of Food Science University of Copenhagen Rolighedsvej 26, 1958 Frederiksberg C, Denmark ${ }^{1}$

${ }^{2}$ COPSAC, Copenhagen Prospective Studies on Asthma in Childhood, Herlev and Gentofte Hospital, University of Copenhagen, Copenhagen, Denmark

\section{Correspondence:}

*Bekzod Khakimov, E-mail: bzo@food.ku.dk; Tel.: +45-2887-4454

*Søren Balling Engelsen, E-mail: se@ @ood.ku.dk; Tel.: +45-3533-3205 


\section{Supporting Information}

\section{Table of content}

Figure S1. ANOVA results from the permutation test $(N=2400)$ performed by randomly selecting 40 subjects out of 165 elderly subjects and comparing with the data of the 40 young subjects.

Figure S2. PC1 versus PC2 scores and loadings plot of the PCA model developed on absolute concentration fecal metabolomics data from the elderly group combined with quality control fecal pool samples.

Figure S3. PC1 versus PC2 scores and loadings plot of the PCA model developed on absolute concentration fecal metabolomics data from the young and elderly group globally and separately.

Figure S4. PLS-DA permutation test developed on absolute concentration fecal metabolomics data from the elderly group.

Figure S5. Scatter plots of selected correlations between sex dependent metabolites and macronutrient intake variables for dietary stratification classes.

Figure S6. Boxplots of three selected metabolites include valeric acid, isovaleric acid and propionic acid after comparing their concentrations in males and females separately.

Table S1. A list of metabolites identified from the ${ }^{1} \mathrm{H}$ NMR spectra of fecal samples analyzed in this study.

Table S2. ANOVA results on absolute concentration fecal metabolomics data including 30 metabolites developed on four sex- and age-specific cases.

Table S3. The explained variation of PCA models developed on absolute concentration fecal metabolomics data including 30 selected metabolites in four sex- and age- stratified datasets.

Table S4. The intake of the five macronutrients (protein, fat, sugars, starch and fiber) in 144 elderly subjects, the unit is g.day/BW (BW = body weight).

Table S5. ANOVA results on the 36 fecal metabolites comparing High Consumption (HC) versus Low Consumption (LC) for the five selected dietary variables: protein, fat, sugars, starch and fiber intakes.

Table S6. ANOVA results on the 16 fecal metabolites from total 36 metabolites calculated on sex- stratified High Consumption (HC) versus Low Consumption (LC) groups for the selected dietary variables. 
Figure S1. ANOVA results from the permutation test $(N=2400)$ performed by randomly selecting 40 subjects out of 165 elderly subjects and comparing with the data of the 40 young subjects. The confidence of the significance of each metabolite have also been reported in the figures.
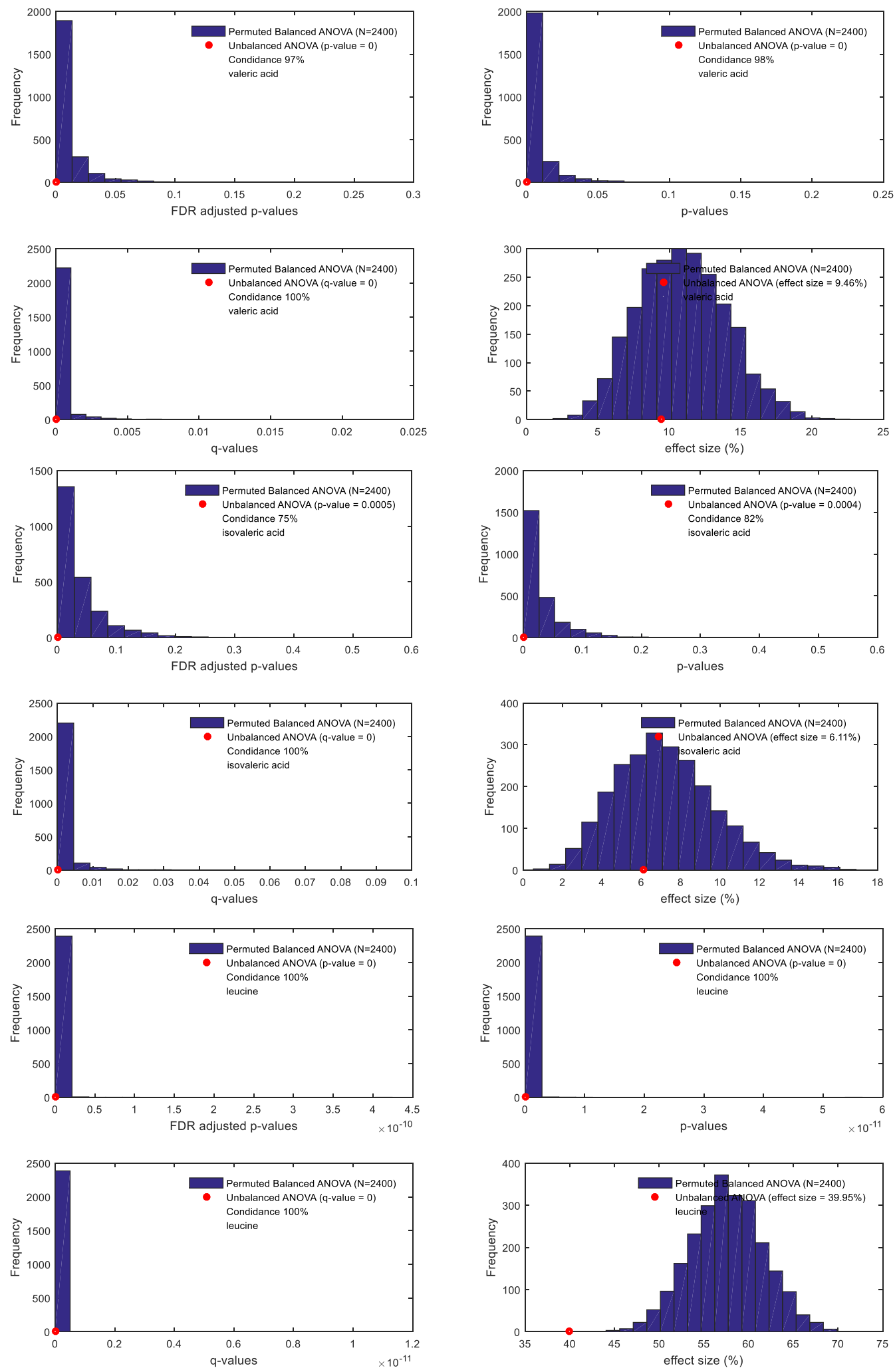

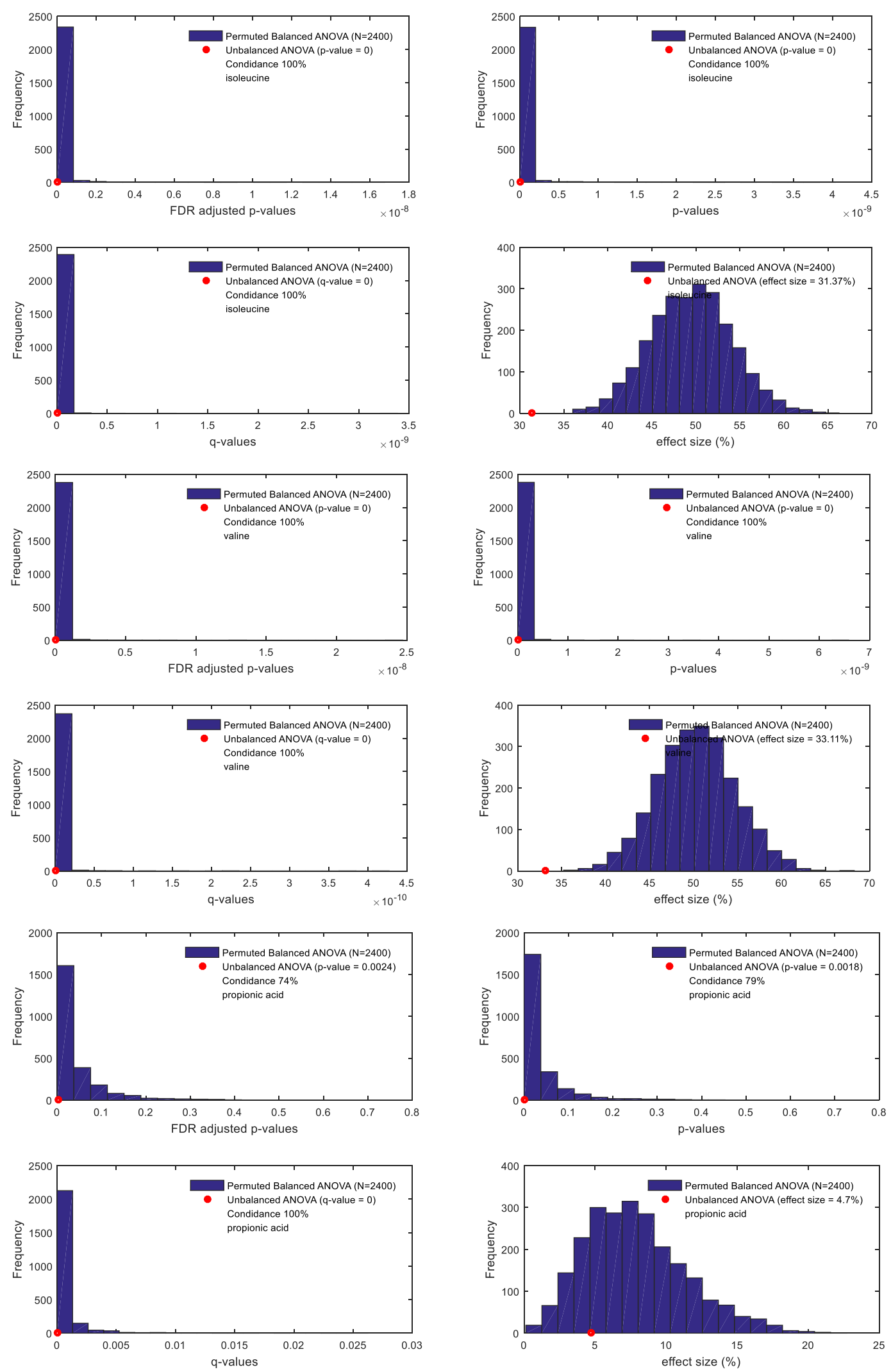

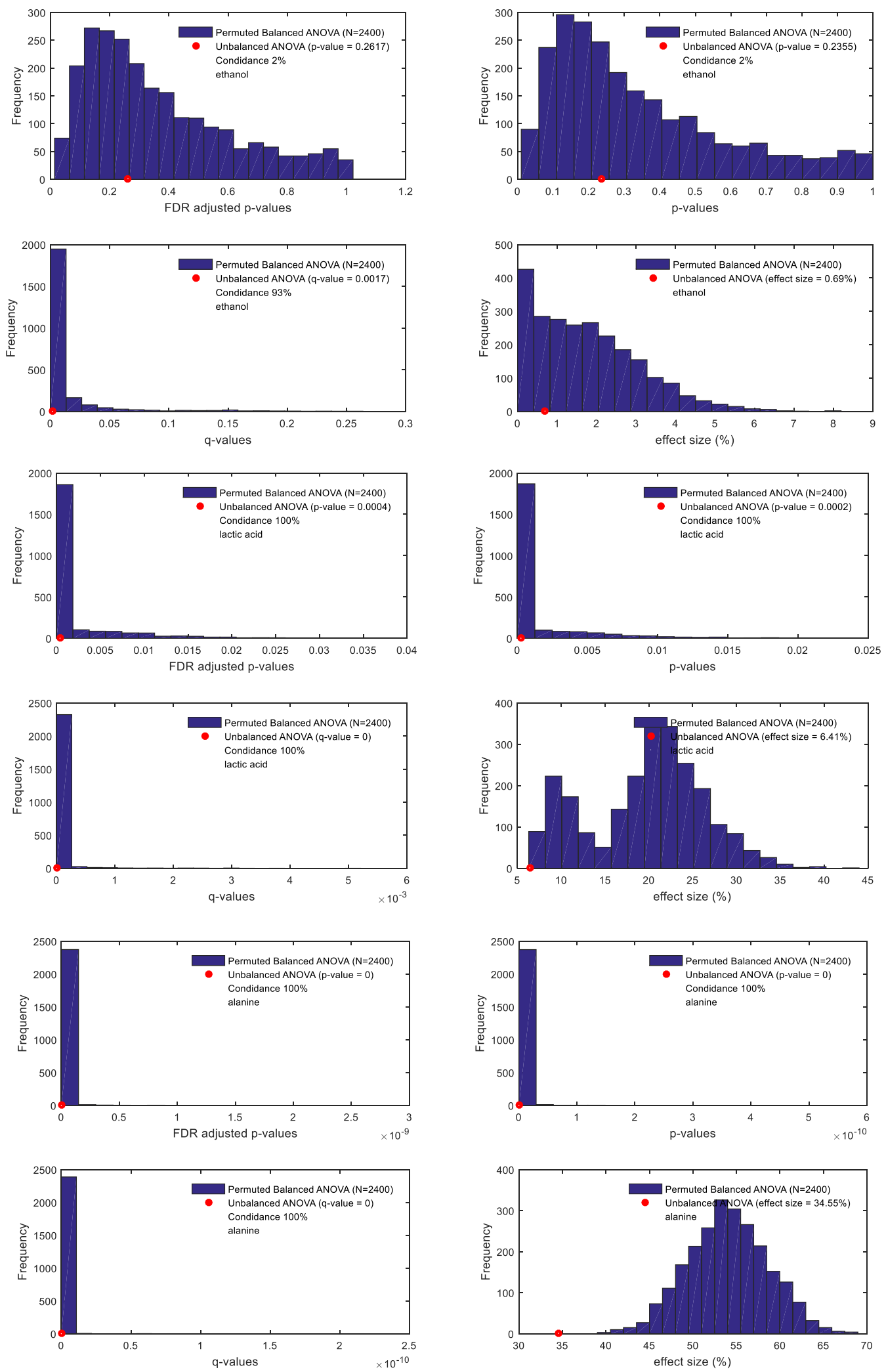

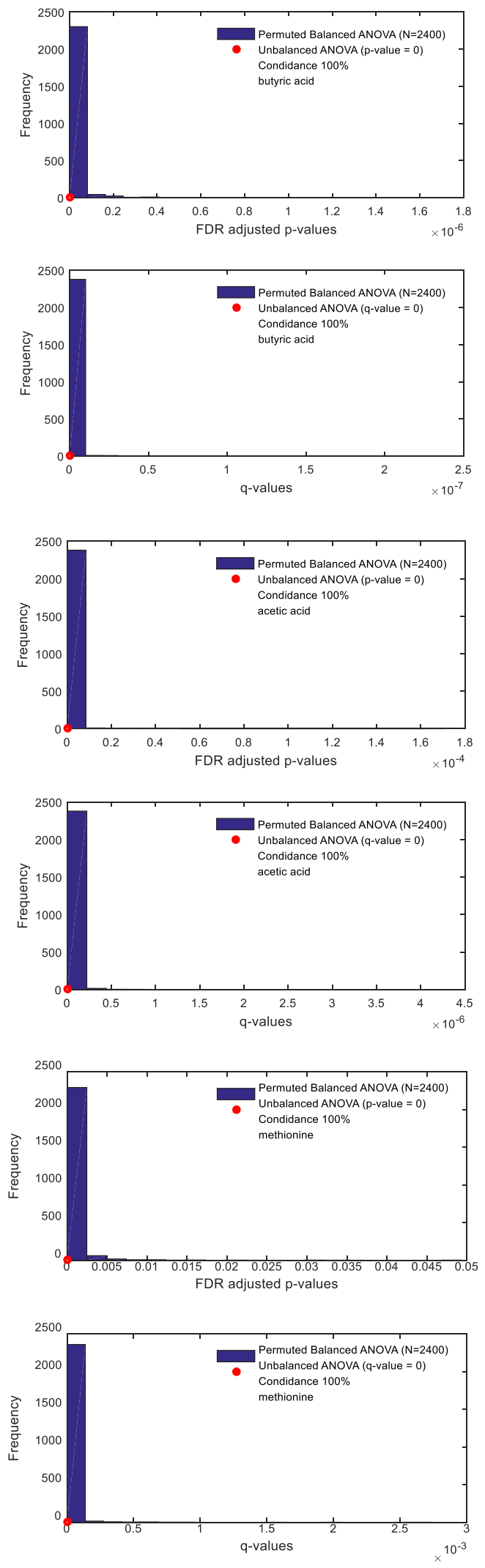
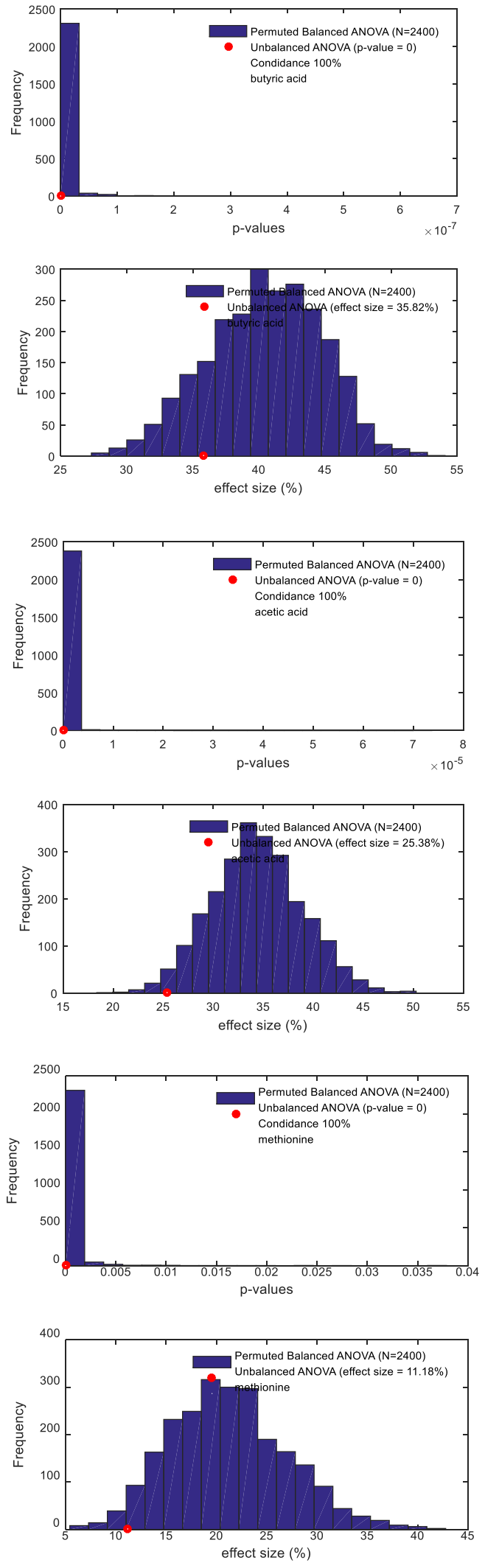

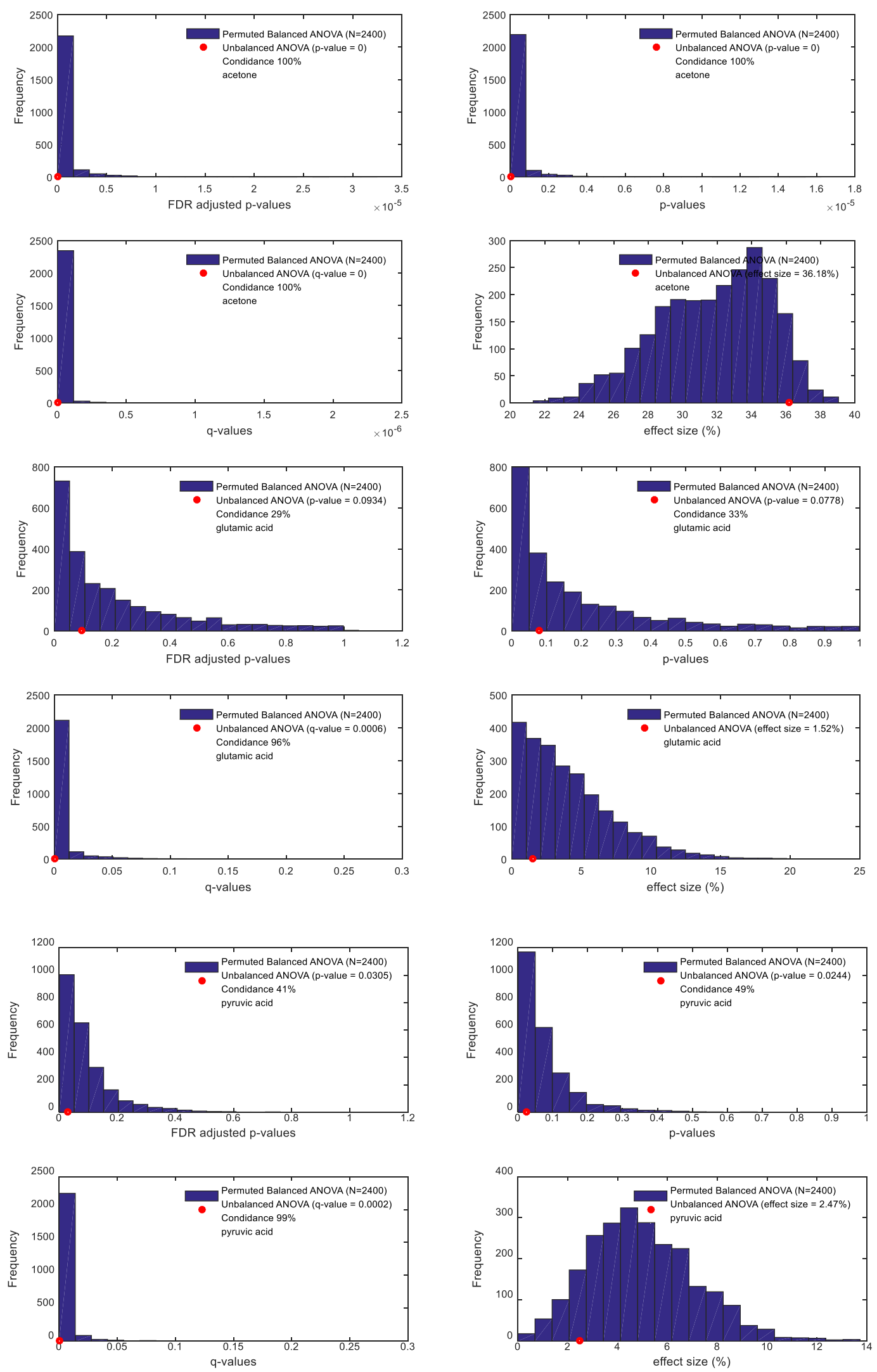

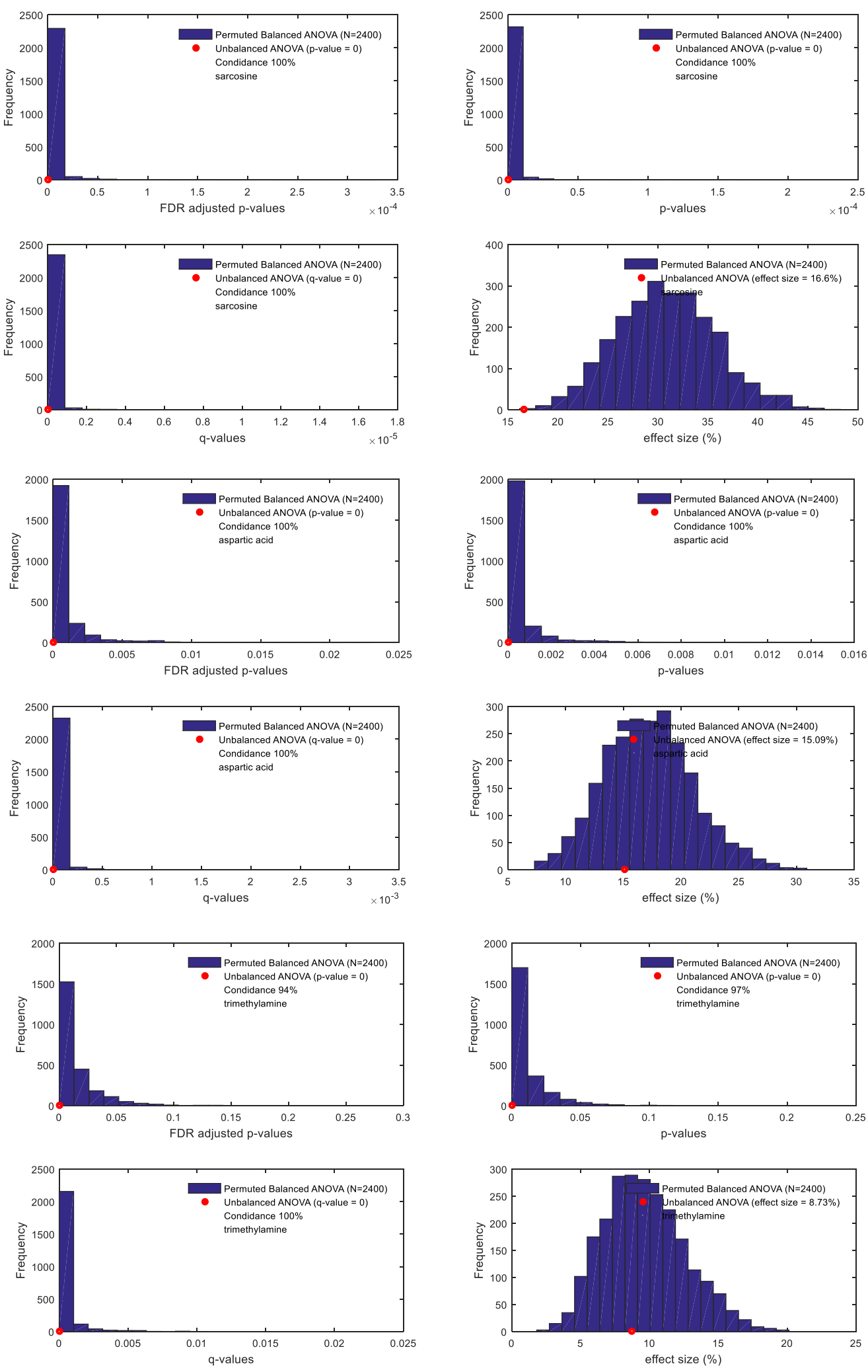

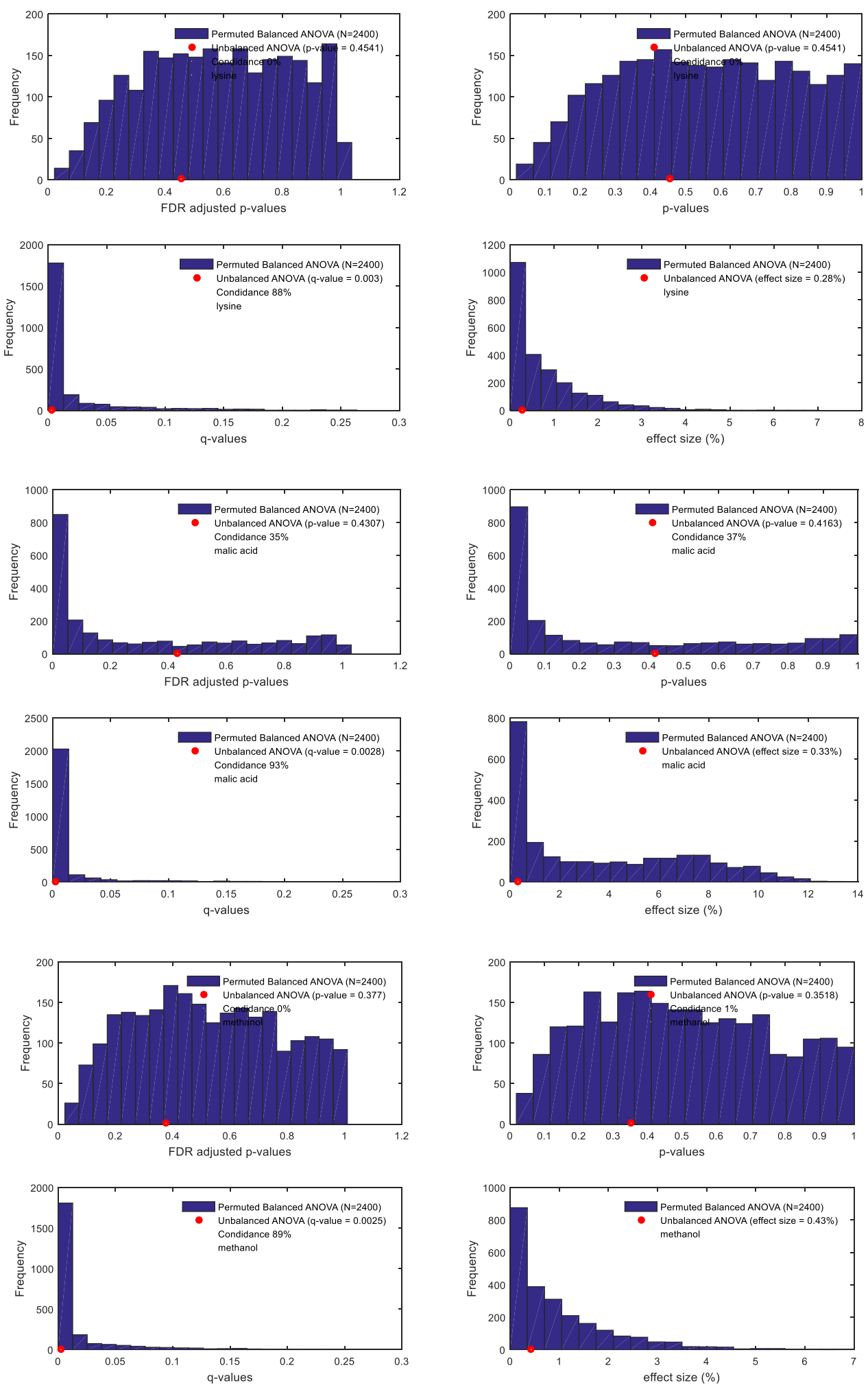

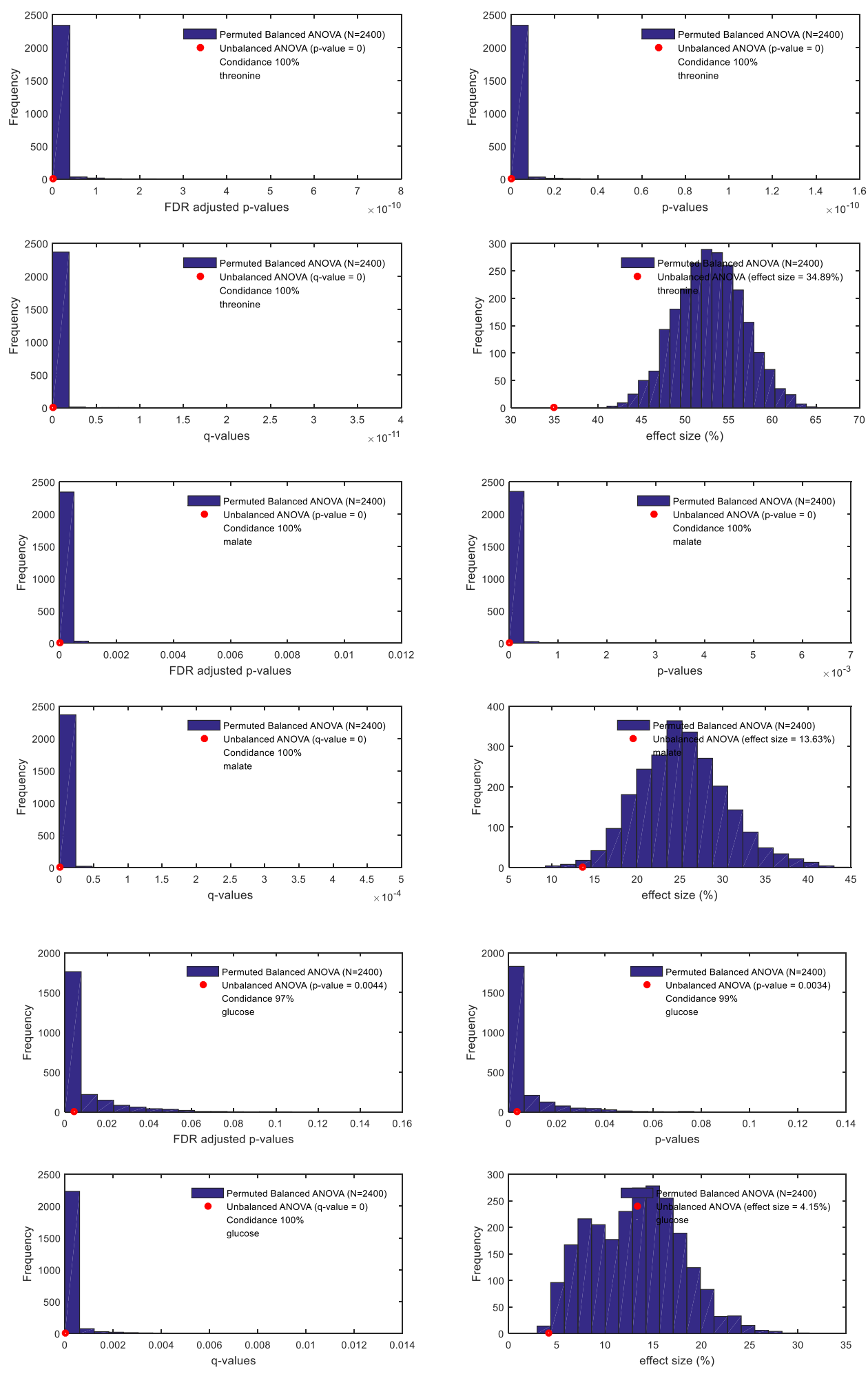

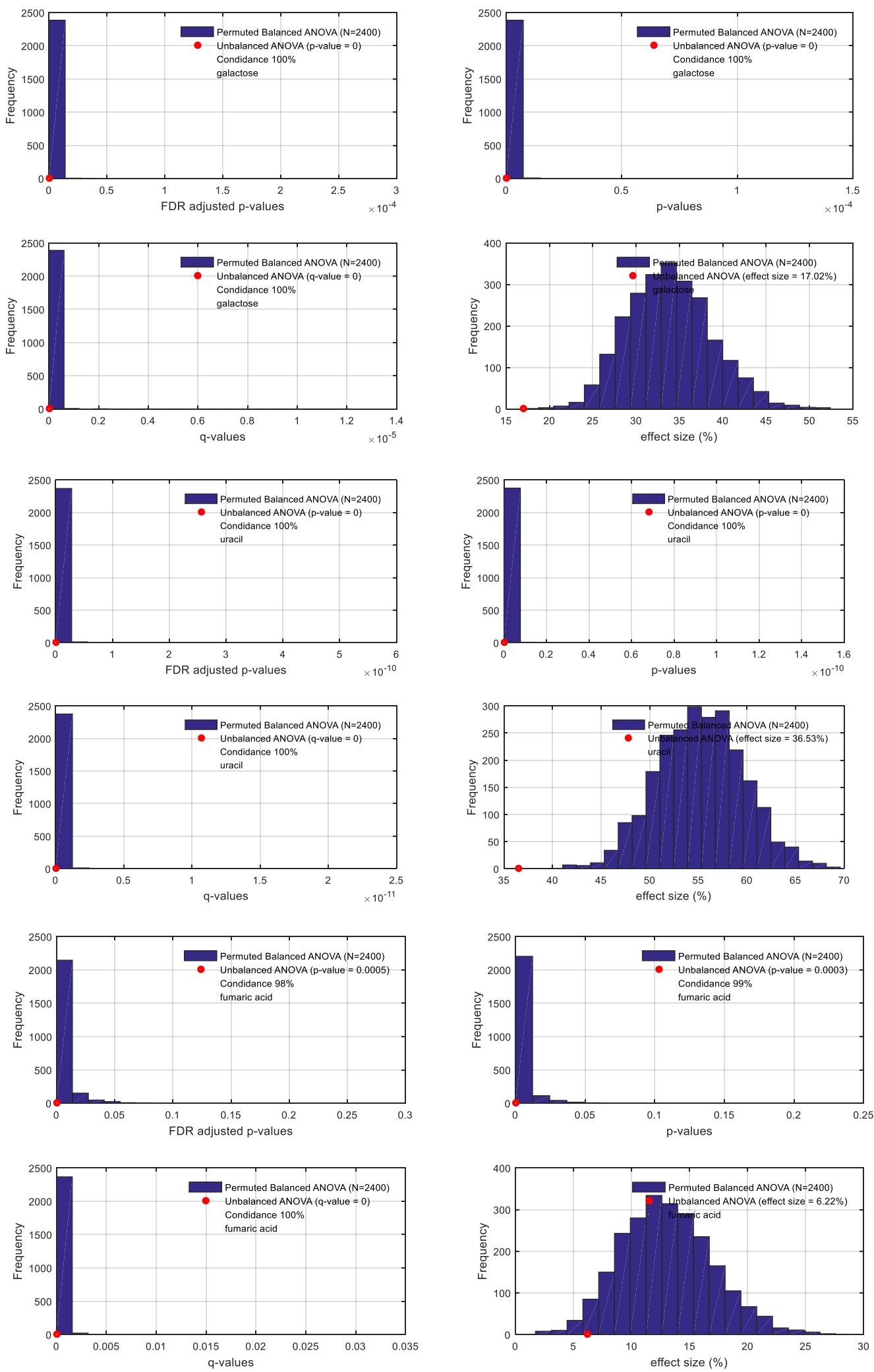

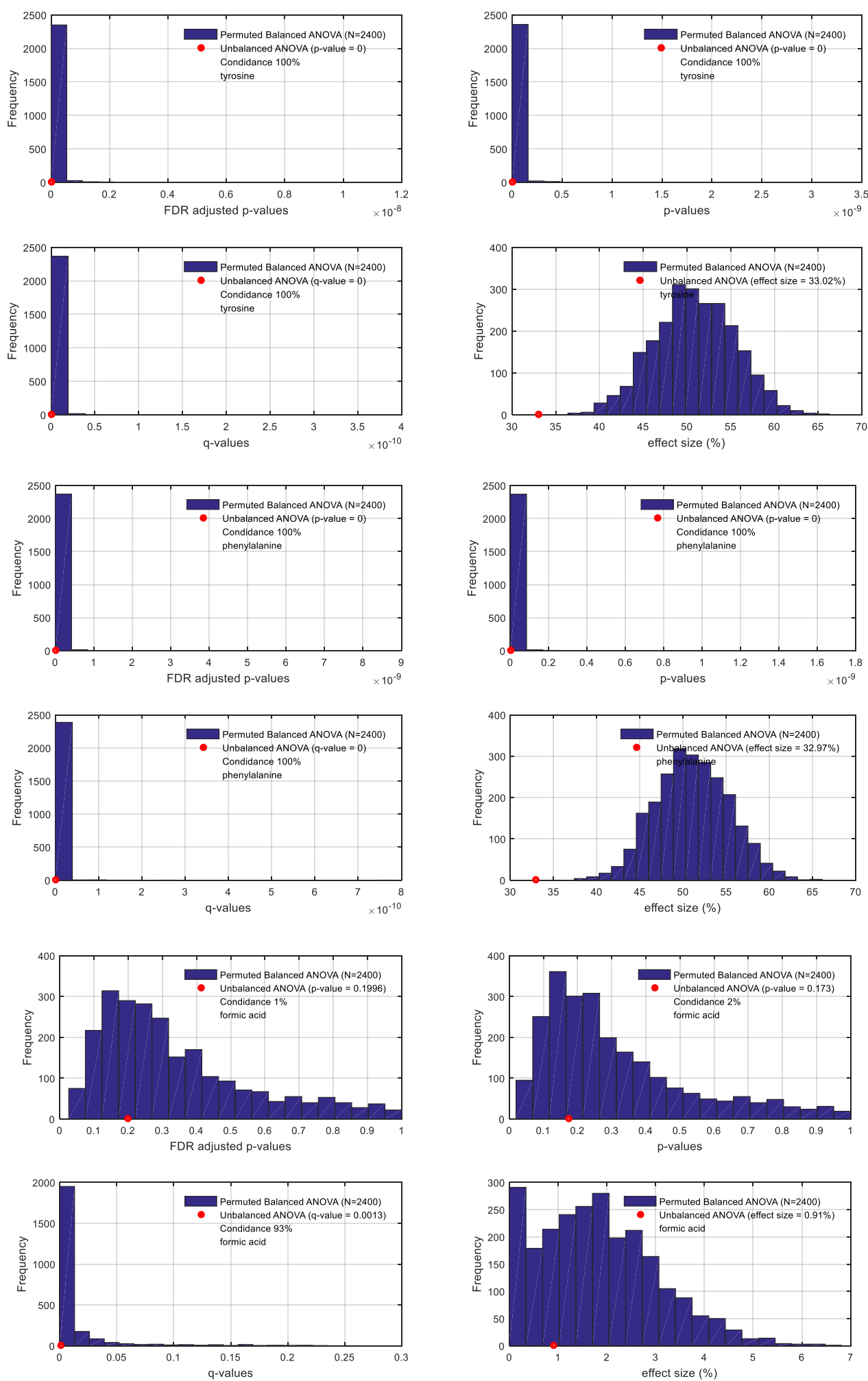

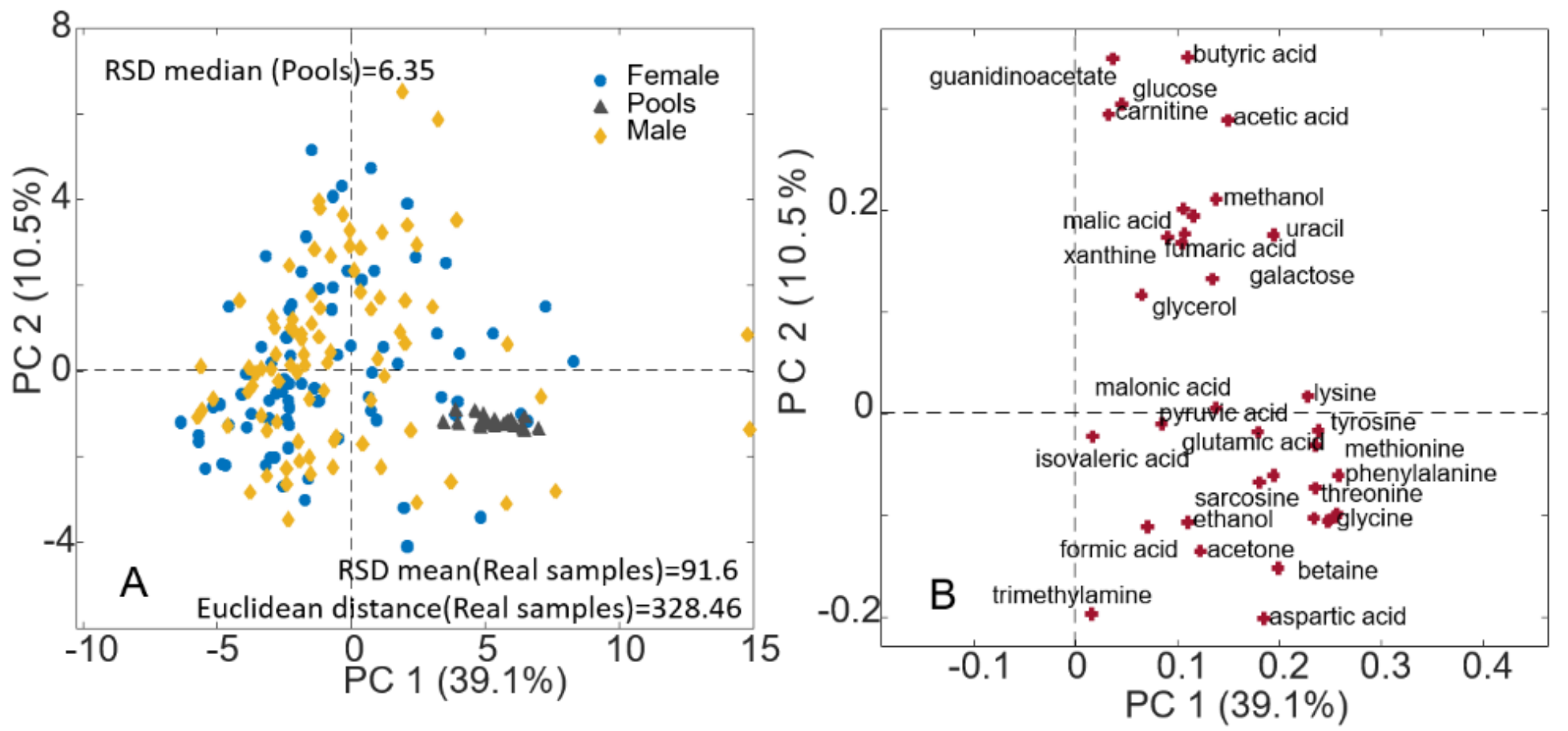

Figure S2. PC1 versus PC2 scores and loadings plot of the PCA model developed on absolute concentration fecal metabolomics data from the elderly group combined with quality control fecal pool samples. Scores plot (A) and loadings plot (B) highlighting sex-related clustering on 185 faecal samples (165 from elderly subjects and 20 pooled samples), and the corresponding metabolites responsible for the observed clustering, respectively. For each PC, the explained variance is given in parenthesis. The mean RSD for pools and real samples and Euclidean distance are reported in the Figure. 

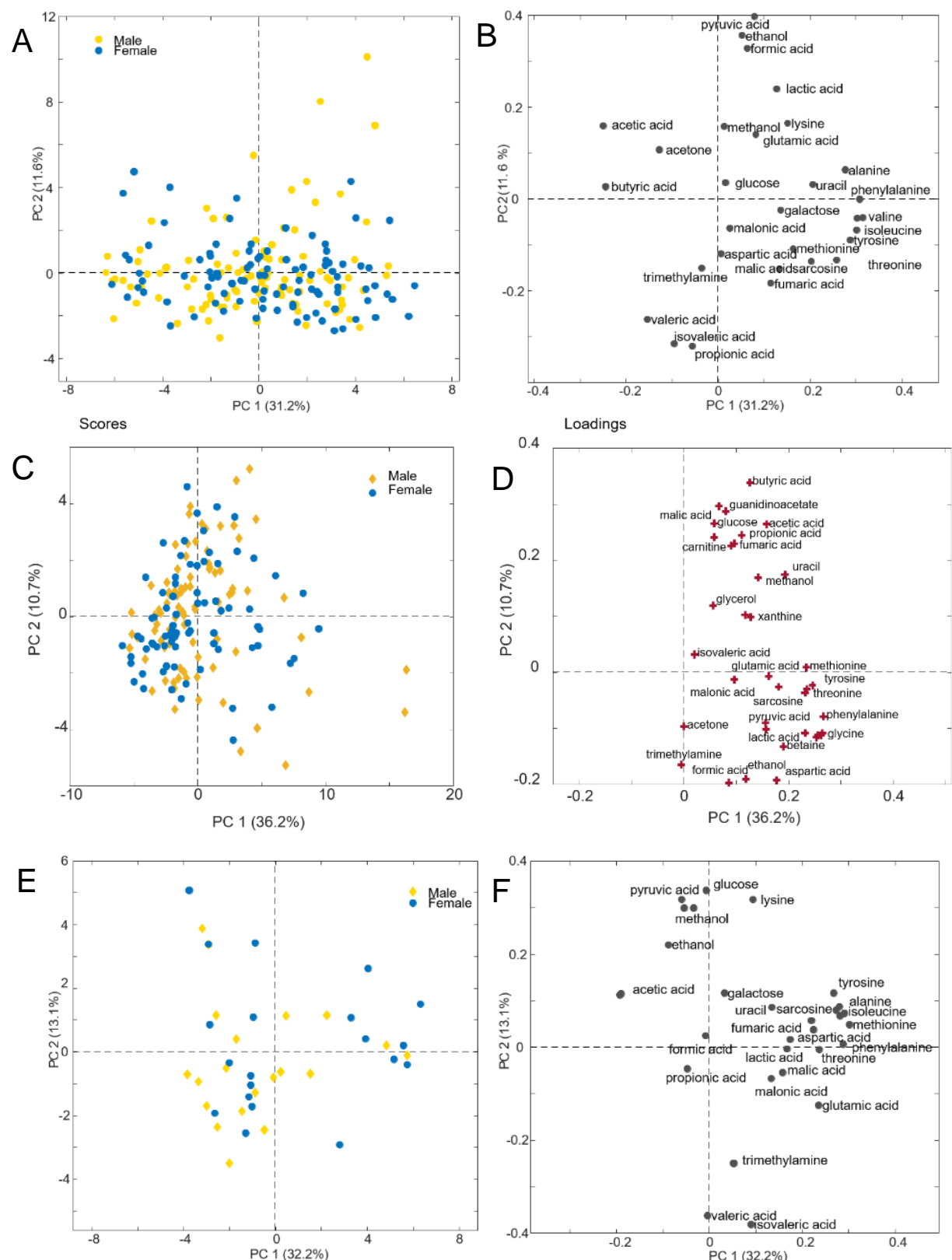

Figure S3. PC1 versus PC2 scores and loadings plot of the PCA model developed on absolute concentration fecal metabolomics data from the young and elderly group globally and separately. PCA Scores plot (A) and loadings plot (B) depicting sex differences between males and females on the global data (205 samples and 30 common variables), scores plot (C) and loadings plot (D) depicting sex differences in the elderly subjects (165 samples and 36 variables) and scores plot (E) and loadings plot (F) depicting sex differences in the young subjects (40 samples and 30 common variables). For each PC, the explained variance is given in parenthesis. 

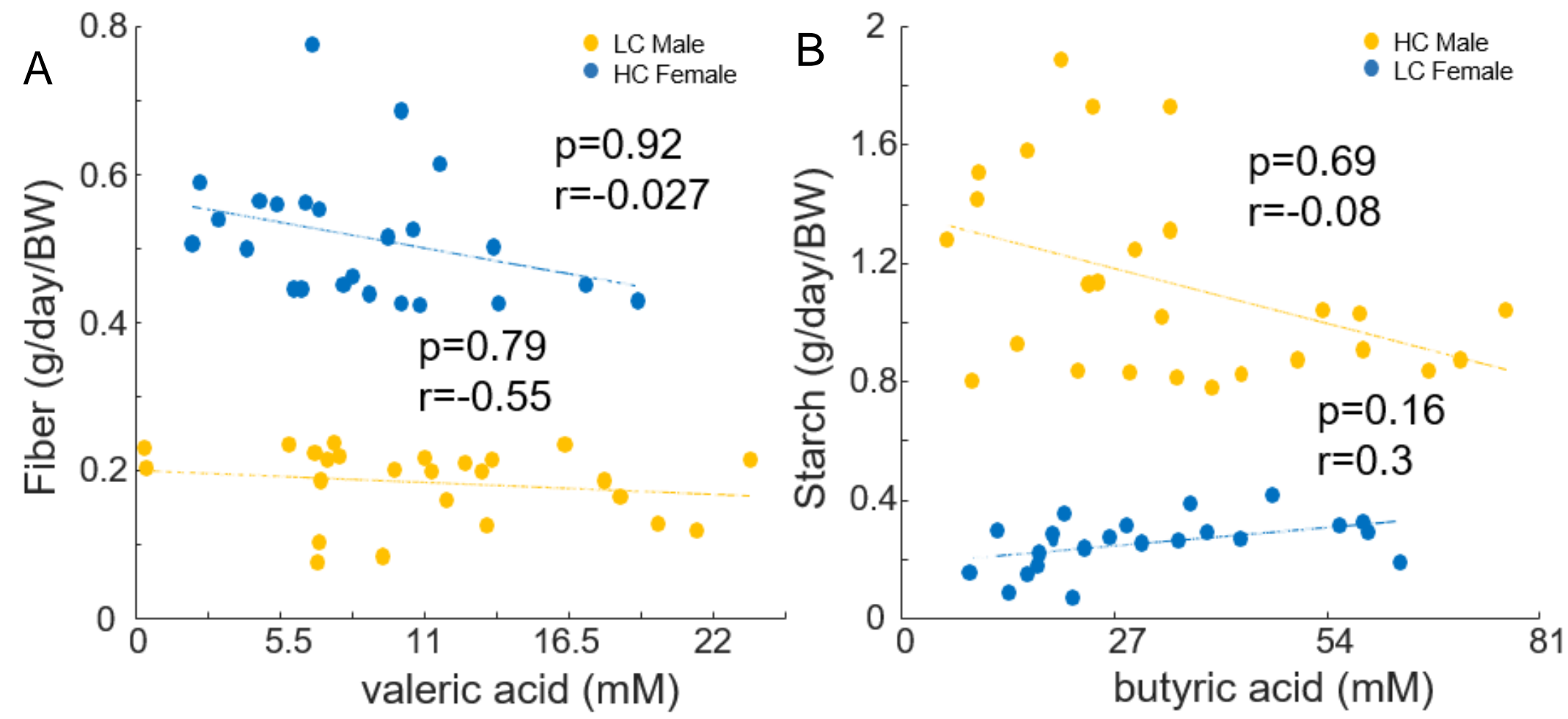

Figure S5. Scatter plots of selected correlations between sex dependent metabolites and macronutrient intake variables for dietary stratification classes of $\mathrm{HC}$ female vs. LC male (A) and HC males vs. LC female (B). LC = low consumption of the macronutrient, $\mathrm{HC}=$ high consumption of the macronutrient, $\mathrm{BW}=$ Body Weight. (144 elderly subjects were employed for the analysis). 

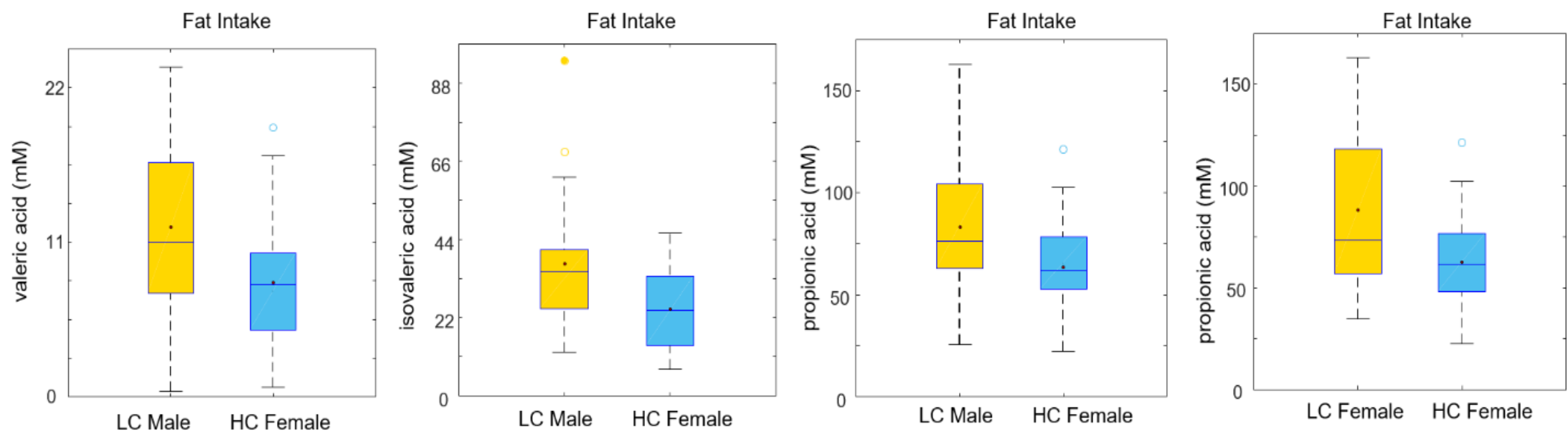

Figure S6. Boxplots of three selected metabolites include valeric acid, isovaleric acid and propionic acid after comparing their concentrations in males and females separately. LC: low consumption of dietary fat, HC: high consumption of dietary fat. 165 elderly subjects were employed in the calculation. The lines and dots inside the boxes represent the median and average of the concentrations of the metabolites, respectively. 
Table S1. A list of metabolites identified from the ${ }^{1} \mathrm{H}$ NMR spectra of fecal samples analyzed in this study. p-value and effect size are obtained from ANOVA for the age and sex effect. E/Y = Elderly/Young; M/F = Male/Female. Normal concentration of metabolites in the human body is reported from the online database HMDB. * Significant p-values which have passed Monte Carlo test. (SCFA= short chain fatty acids; AA = amino acids; $\mathrm{OA}=$ organic acids; $\mathrm{CBH}=$ Carbohydrate; $\mathrm{NA}=$ nucleic acids).

\begin{tabular}{|c|c|c|c|c|c|c|c|c|c|c|c|c|}
\hline \multirow{3}{*}{$\begin{array}{c}\text { No. } \\
1\end{array}$} & \multirow[b]{2}{*}{ Name } & \multirow[b]{2}{*}{ Subgroup } & \multirow{2}{*}{\multicolumn{2}{|c|}{ ppm range }} & \multicolumn{2}{|c|}{$\begin{array}{l}\text { Fold change* } \\
\text { (Mean) }\end{array}$} & \multicolumn{2}{|c|}{ p-value } & \multicolumn{2}{|c|}{ Effect size (\%) } & \multirow[b]{2}{*}{$\begin{array}{c}\text { Mean( } \pm \text { SD }) \\
\text { nmol/g }\end{array}$} & \multirow{2}{*}{$\begin{array}{c}\text { Normal range } \\
\text { (from HMDB) } \\
\text { nmol/g }\end{array}$} \\
\hline & & & & & $\begin{array}{c}\text { age } \\
(\mathbf{E} / \mathbf{Y})\end{array}$ & $\begin{array}{c}\mathbf{s e x} \\
(\mathbf{M} / \mathbf{F})\end{array}$ & age & sex & age & sex & & \\
\hline & valeric acid & SCFA & 0.8954 & 0.8912 & 0.726 & 1.25 & 1.32E-05 & 0.02 & 9.17 & 3.18 & $51354 \pm 25488$ & $600-4300$ \\
\hline 2 & isovaleric acid & SCFA & 0.9243 & 0.9173 & 0.738 & 1.28 & 4.37E-04 & 0.01 & 6.048 & 4 & $153441 \pm 72522$ & $70-3230$ \\
\hline 3 & leucine & AA & 0.98 & 0.949 & 2.107 & 1.06 & $1.50 \mathrm{E}-23$ & 0.45 & 40.972 & 0.35 & $94084 \pm 39599$ & $50-8209$ \\
\hline 4 & isoleucine & AA & 1.026 & 1.002 & 1.94 & 1.11 & $9.13 \mathrm{E}-19$ & 0.88 & 32.838 & 0.01 & $153853 \pm 62469$ & $160-89398$ \\
\hline 5 & valine & AA & 1.043 & 1.032 & 1.981 & 1.03 & $1.60 \mathrm{E}-19$ & 0.64 & 34.231 & 0.14 & $123250 \pm 51010$ & $250-100102$ \\
\hline 6 & propionic acid & SCFA & 1.08 & 1.068 & 1.148 & 1.12 & 0.0015 & 0.16 & 4.933 & 1.22 & $400873 \pm 143749$ & $0-1304067$ \\
\hline 7 & ethanol & N.A. & 1.213 & 1.18 & 1.388 & 1.52 & $6.16 \mathrm{E}-06$ & 0.03 & 9.865 & 2.86 & $20624 \pm 31724$ & $0-1025576$ \\
\hline 8 & lactic acid & SCFA & 1.345 & 1.32 & 1.461 & 1.11 & $6.54 \mathrm{E}-08$ & 0.19 & 13.848 & 1.03 & $73020 \pm 98320$ & $0-35043$ \\
\hline 9 & alanine & AA & 1.503 & 1.466 & 2.182 & 1.01 & $1.94 \mathrm{E}-20$ & 0.34 & 35.825 & 0.56 & $105701 \pm 46165$ & $137-12245$ \\
\hline 10 & butyric acid & SCFA & 1.595 & 1.531 & 0.539 & 1.28 & $1.57 \mathrm{E}-19$ & 0.05 & 34.358 & 2.36 & $172515 \pm 93266$ & $0-56788$ \\
\hline 11 & acetic acid & OA & 1.932 & 1.916 & 0.782 & 1.18 & $7.71 \mathrm{E}-13$ & 0.2 & 23.221 & 0.98 & $660150 \pm 252517$ & $12800-103400$ \\
\hline 12 & methionine & AA & 2.144 & 2.138 & 1.617 & 1.26 & $7.26 \mathrm{E}-07$ & 0.56 & 11.756 & 0.21 & $38094 \pm 21949$ & $0-35118$ \\
\hline 13 & acetone & N.A. & 2.239 & 2.235 & 0.519 & 0.91 & $2.60 \mathrm{E}-08$ & 0.32 & 14.701 & 0.61 & $960 \pm 1560$ & $0-337126$ \\
\hline 14 & glutamic acid & AA & 2.377 & 2.341 & 1.306 & 1.12 & 0.0662 & 0.36 & 1.681 & 0.5 & $45299 \pm 32633$ & $484+/-314$ \\
\hline 15 & pyruvic acid & $\mathrm{OA}$ & 2.415 & 2.402 & 1.494 & 1.05 & 7.57E-09 & 0.15 & 15.8 & 1.27 & $38677 \pm 59377$ & $363-91942$ \\
\hline 16 & aspartic acid & $\mathrm{AA}$ & 2.706 & 2.701 & 1.892 & 0.95 & $1.57 \mathrm{E}-09$ & 0.58 & 17.26 & 0.19 & $27814 \pm 16328$ & $130+/-120$ \\
\hline 17 & sarcosine & AA & 2.761 & 2.753 & 0.596 & 1.04 & $1.30 \mathrm{E}-08$ & 0.52 & 15.318 & 0.26 & $2365 \pm 1097$ & N.A. \\
\hline 18 & trimethylamine & N.A. & 2.869 & 2.86 & 0.452 & 0.48 & $2.02 \mathrm{E}-05$ & 0.11 & 8.774 & 1.55 & $1440 \pm 1410$ & N.A. \\
\hline 19 & lysine & AA & 3.057 & 3.005 & 1.061 & 1.06 & 0.3826 & 0.27 & 0.376 & 0.76 & $56583 \pm 26845$ & $540+/-410$ \\
\hline 20 & malonic acid & $\mathrm{OA}$ & 3.14 & 3.129 & 1.253 & 0.92 & $9.57 \mathrm{E}-04$ & 0.83 & 5.3 & 0.03 & $4320 \pm 7560$ & N.A. \\
\hline 21 & methanol & N.A. & 3.371 & 3.36 & 1.368 & 0.85 & $3.38 \mathrm{E}-06$ & 0.7 & 10.413 & 0.09 & $6502 \pm 4590$ & N.A. \\
\hline 22 & carnitine & N.A. & 3.423 & 3.4161 & N.A. & 1.18 & N.A. & 0.86 & N.A. & 0.02 & $3887 \pm 8319$ & $8+/-3$ \\
\hline 23 & glycine & AA & 3.572 & 3.561 & N.A. & 0.97 & N.A. & 0.61 & N.A. & 0.16 & $45312 \pm 18252$ & $400+/-290$ \\
\hline 24 & glycerol & N.A. & 3.652 & 3.645 & N.A. & 0.98 & N.A. & 0.96 & N.A. & 0 & $51975 \pm 35043$ & $6000-22911$ \\
\hline 25 & $\begin{array}{c}\text { guanidinoacetic } \\
\text { acid } \\
\end{array}$ & N.A. & 3.802 & 3.799 & N.A. & 1.54 & N.A. & 0.53 & N.A. & 0.24 & $368 \pm 402$ & N.A. \\
\hline 26 & betaine & N.A. & 3.905 & 3.9022 & N.A. & 1.13 & N.A. & 0.67 & N.A. & 0.11 & $8460 \pm 5460$ & N.A. \\
\hline 27 & threonine & AA & 4.286 & 4.237 & 2.265 & 1.07 & $1.25 \mathrm{E}-20$ & 0.71 & 36.278 & 0.08 & $22633 \pm 10483$ & $10-540$ \\
\hline 28 & malic acid & $\mathrm{OA}$ & 4.327 & 4.295 & 2.27 & 0.91 & 5.75E-08 & 0.76 & 14.073 & 0.06 & $9435 \pm 95490$ & $0-921$ \\
\hline 29 & glucose & $\mathrm{CH}$ & 5.249 & 5.234 & 1.562 & 1.43 & 3.34E-09 & 0.67 & 16.536 & 0.11 & $45359 \pm 73324$ & $700-18271$ \\
\hline
\end{tabular}




\begin{tabular}{|c|c|c|c|c|c|c|c|c|c|c|c|c|}
\cline { 5 - 12 } 30 & galactose & CH & 5.281 & 5.266 & 1.619 & 0.78 & $2.25 \mathrm{E}-09$ & 0.44 & 16.916 & 0.36 & $5897 \pm 4374$ & $746+/-394$ \\
\hline 31 & uracil & NA & 5.823 & 5.787 & 2.336 & 1.06 & $1.87 \mathrm{E}-21$ & 0.54 & 37.7 & 0.23 & $10890 \pm 4020$ & N.A. \\
\hline 32 & fumaric acid & OA & 6.527 & 6.521 & 1.756 & 0.92 & $2.80 \mathrm{E}-04$ & 0.72 & 6.468 & 0.08 & $1875 \pm 1260$ & $84+/-50$ \\
\hline 33 & tyrosine & AA & 7.215 & 7.176 & 2.138 & 1.03 & $4.11 \mathrm{E}-19$ & 0.88 & 33.522 & 0.01 & $20656 \pm 8533$ & $10-1200$ \\
\hline 34 & phenylalanine & AA & 7.349 & 7.316 & 1.855 & 1.03 & $7.29 \mathrm{E}-19$ & 0.73 & 33.063 & 0.07 & $26792 \pm 11519$ & $0-1200$ \\
\hline 35 & xanthine & N.A. & 7.93 & 7.91 & N.A. & 1.04 & N.A. & 0.72 & N.A. & 0.08 & $1875 \pm 1057$ & N.A. \\
\hline 36 & formic acid & N.A. & 8.47 & 8.44 & 1.566 & 1.05 & $1.23 \mathrm{E}-07$ & 0.13 & 13.279 & 1.4 & $3000 \pm 7080$ & N.A. \\
\hline
\end{tabular}


Table S2. ANOVA results on absolute concentration fecal metabolomics data including 30 metabolites developed on four sex- and age-specific cases, case $1=$ Young Females vs. Elderly Females (A), case $2=$ Young Males vs. Elderly Males (B), case $3=$ Young Females vs. Elderly Males (C) and case $4=$ Young Males vs. Elderly Females (D). * Significant p-values which have passed Monte Carlo test.

Elderly Females versus Young Females

\begin{tabular}{llccc}
\hline order & Name & p-value (FDR) & Effect Size $(\%)$ & Fold change Mean(E/Y) \\
\hline 1 & valeric acid & 0.0087 & 7.131 & 0.750 \\
2 & isovaleric acid & 0.0025 & 9.490 & 0.675 \\
3 & leucine & $5.2 \mathrm{E}-11$ & 39.144 & 1.972 \\
4 & isoleucine & $1.68 \mathrm{E}-09$ & 32.884 & 1.883 \\
5 & valine & $4.9 \mathrm{E}-09$ & 31.272 & 1.861 \\
6 & propionic acid & 0.0025 & 9.630 & 1.198 \\
7 & ethanol & 0.0109 & 6.672 & 1.288 \\
8 & lactic acid & 0.0011 & 11.239 & 1.389 \\
9 & alanine & $2.7 \mathrm{E}-10$ & 36.191 & 2.058 \\
10 & butyric acid & $2.7 \mathrm{E}-10$ & 36.429 & 0.531 \\
11 & acetic acid & 0.000017 & 18.880 & 0.794 \\
12 & methionine & 0.0074 & 7.551 & 0.519 \\
13 & acetone & 0.0000418 & 17.176 & 1.138 \\
14 & pyruvic acid & 0.0005 & 12.629 & 1.397 \\
15 & sarcosine & 0.0000319 & 17.742 & 1.871 \\
16 & aspartic acid & 0.00000658 & 20.538 & 1.007 \\
17 & trimethylamine & 0.0087 & 7.147 & 1.168 \\
18 & methanol & 0.0025 & 9.563 & 1.307 \\
19 & threonine & $9.28 \mathrm{E}-10$ & 33.858 & 2.048 \\
20 & malic acid & 0.0002 & 14.724 & 2.343 \\
21 & glucose & 0.0002 & 14.070 & 1.433 \\
22 & galactose & 0.0001 & 16.283 & 1.546 \\
23 & uracil & $5.11 \mathrm{E}-11$ & 39.383 & 2.281 \\
24 & fumaric acid & 0.0110 & 6.601 & 1.806 \\
25 & tyrosine & $4.49 \mathrm{E}-10$ & 35.250 & 2.093 \\
26 & phenylalanine & $6.55 \mathrm{E}-10$ & 34.519 & 1.803 \\
27 & formic acid & 0.0012 & 10.988 & \\
\hline & & & & \\
\end{tabular}

Elderly Males versus Young Males

\begin{tabular}{llccc}
\hline order & Name & $\begin{array}{c}\text { p-value } \\
(\text { FDR })\end{array}$ & $\begin{array}{c}\text { Effect Size } \\
(\%)\end{array}$ & Fold change Mean(E/Y) \\
\hline 1 & valeric acid & 0.0004 & 11.872 & 0.703
\end{tabular}




\begin{tabular}{|c|c|c|c|c|}
\hline 2 & leucine & $5.59 \mathrm{E}-13$ & 43.576 & 2.274 \\
\hline 3 & isoleucine & $3.01 \mathrm{E}-10$ & 38.622 & 2.012 \\
\hline 4 & valine & $1.50 \mathrm{E}-11$ & 22.491 & 2.134 \\
\hline 5 & ethanol & 0.0001 & 13.925 & 1.513 \\
\hline 6 & lactic acid & $1.55 \mathrm{E}-05$ & 17.941 & 1.555 \\
\hline 7 & alanine & $8.73 \mathrm{E}-11$ & 35.900 & 2.328 \\
\hline 8 & butyric acid & $4.08 \mathrm{E}-10$ & 33.561 & 0.545 \\
\hline 9 & acetic acid & $1.46 \mathrm{E}-08$ & 28.397 & 0.770 \\
\hline 10 & methionine & $1.60 \mathrm{E}-05$ & 17.674 & 1.785 \\
\hline 11 & acetone & 0.0003 & 12.538 & 0.523 \\
\hline 12 & glutamic acid & 0.0503 & 38.345 & 1.513 \\
\hline 13 & pyruvic acid & 4.22E-06 & 20.168 & 1.618 \\
\hline 14 & sarcosine & $2.43 \mathrm{E}-05$ & 16.932 & 1.916 \\
\hline 15 & aspartic acid & 0.0008 & 10.733 & 0.662 \\
\hline 16 & trimethylamine & 0.0008 & 10.778 & 0.393 \\
\hline 17 & lysine & 0.0012 & 9.987 & 1.368 \\
\hline 18 & methanol & 0.0004 & 12.091 & 1.448 \\
\hline 19 & threonine & $9.11 \mathrm{E}-12$ & 39.678 & 2.558 \\
\hline 20 & malic acid & 0.0002 & 13.406 & 2.202 \\
\hline 21 & glucose & 4.89E-06 & 19.815 & 1.733 \\
\hline 22 & galactose & $9.17 \mathrm{E}-06$ & 18.744 & 1.717 \\
\hline 23 & uracil & $7.12 \mathrm{E}-11$ & 36.421 & 2.398 \\
\hline 24 & fumaric acid & 0.0095 & 6.583 & 1.709 \\
\hline 25 & tyrosine & 4.20E-10 & 33.355 & 2.198 \\
\hline 26 & phenylalanine & $5.89 \mathrm{E}-10$ & 32.773 & 1.918 \\
\hline 27 & formic acid & $2.53 \mathrm{E}-05$ & 16.776 & 1.639 \\
\hline
\end{tabular}

Elderly Males versus Young Females

\begin{tabular}{llrrc}
\hline order & Name & $\begin{array}{c}\text { p-value } \\
\text { (FDR) }\end{array}$ & $\begin{array}{c}\text { Effect Size } \\
(\%)\end{array}$ & Fold change Mean(E/Y) \\
\hline 1 & isovaleric acid & 0.0334 & 4.610 & 0.762 \\
2 & leucine & $2.91 \mathrm{E}-09$ & 33.536 & 1.878 \\
3 & isoleucine & $1.10 \mathrm{E}-07$ & 25.914 & 1.726 \\
4 & valine & $6.77 \mathrm{E}-08$ & 27.244 & 1.734 \\
5 & propionic acid & 0.0040 & 8.433 & 1.216 \\
6 & ethanol & 0.0186 & 5.693 & 1.234 \\
7 & lactic acid & 0.0051 & 7.961 & 1.271 \\
8 & alanine & $1.84 \mathrm{E}-08$ & 29.705 & 1.991 \\
9 & butyric acid & $9.31 \mathrm{E}-08$ & 26.336 & 0.593 \\
10 & acetic acid & $5.94 \mathrm{E}-07$ & 23.169 & 0.785 \\
11 & methionine & 0.0018 & 9.871 & 1.478 \\
12 & acetone & $9.66 \mathrm{E}-07$ & 22.221 & 0.437
\end{tabular}




\begin{tabular}{llrrl}
13 & pyruvic acid & 0.0018 & 9.892 & 1.302 \\
14 & sarcosine & 0.0001 & 14.905 & 1.802 \\
15 & aspartic acid & $7.48 \mathrm{E}-08$ & 26.855 & 0.476 \\
16 & trimethylamine & 0.0003 & 13.071 & 0.359 \\
17 & methanol & 0.0518 & 3.848 & 1.182 \\
18 & threonine & $5.51 \mathrm{E}-08$ & 27.834 & 1.921 \\
19 & malic acid & 0.0003 & 13.233 & 2.197 \\
20 & glucose & 0.0051 & 7.867 & 1.331 \\
21 & galactose & 0.0021 & 9.566 & 1.380 \\
22 & uracil & $4.66 \mathrm{E}-09$ & 32.046 & 2.200 \\
23 & fumaric acid & 0.0210 & 5.424 & 1.619 \\
24 & tyrosine & $1.14 \mathrm{E}-07$ & 25.697 & 1.880 \\
25 & phenylalanine & $2.08 \mathrm{E}-07$ & 24.699 & 1.675 \\
26 & formic acid & 0.0008 & 11.424 & 1.400 \\
\hline
\end{tabular}

Elderly Females versus Young Males

\begin{tabular}{llrrc}
\hline order & Name & \multicolumn{1}{c}{$\begin{array}{c}\text { p-value } \\
\text { (FDR) }\end{array}$} & $\begin{array}{c}\text { Effect Size } \\
(\%)\end{array}$ & Fold change Mean(E/Y) \\
\hline 1 & valeric acid & $2.21 \mathrm{E}-07$ & 25.430 & 0.626 \\
2 & isovaleric acid & 0.0047 & 8.337 & 0.709 \\
3 & leucine & $1.78 \mathrm{E}-14$ & 48.890 & 2.388 \\
4 & isoleucine & $3.39 \mathrm{E}-12$ & 40.609 & 2.193 \\
5 & valine & $1.55 \mathrm{E}-12$ & 41.676 & 2.290 \\
6 & ethanol & 0.0002 & 14.308 & 1.579 \\
7 & lactic acid & $5.29 \mathrm{E}-06$ & 20.245 & 1.698 \\
8 & alanine & $8.28 \mathrm{E}-13$ & 42.562 & 2.406 \\
9 & butyric acid & $7.60 \mathrm{E}-13$ & 43.303 & 0.488 \\
10 & acetic aicd & $8.55 \mathrm{E}-07$ & 23.280 & 0.779 \\
11 & methionine & 0.0002 & 13.639 & 1.783 \\
12 & acetone & 0.0047 & 8.253 & 0.621 \\
13 & pyruvic acid & $1.77 \mathrm{E}-06$ & 22.053 & 1.736 \\
14 & sarcosine & $6.19 \mathrm{E}-06$ & 19.897 & 1.989 \\
15 & aspartic aicd & 0.0255 & 5.166 & 0.765 \\
16 & trimethylamine & 0.0249 & 5.270 & 0.558 \\
17 & malonic acid & 0.0002 & 14.182 & 1.521 \\
18 & methanol & $1.14 \mathrm{E}-05$ & 18.831 & 1.601 \\
19 & threonine & $2.42 \mathrm{E}-13$ & 45.365 & 2.727 \\
20 & malic acid & 0.0001 & 14.895 & 2.349 \\
21 & glucose & $4.92 \mathrm{E}-08$ & 27.886 & 1.866 \\
22 & galactose & $2.21 \mathrm{E}-07$ & 25.520 & 1.924 \\
23 & uracil & $4.37 \mathrm{E}-13$ & 44.256 & 2.487 \\
24 & fumaric acid & 0.0064 & 7.644 & 1.907
\end{tabular}




\begin{tabular}{lllll}
25 & tyrosine & $8.17 \mathrm{E}-13$ & 42.755 & 2.446 \\
26 & phenylalanine & $8.17 \mathrm{E}-13$ & 42.867 & 2.065 \\
27 & formic acid & $8.39 \mathrm{E}-05$ & 15.511 & 1.764 \\
\hline
\end{tabular}


Table S3. The explained variation of PCA models developed on absolute concentration faecal metabolomics data including 30 selected metabolites in four sex- and age- stratified datasets, Young Females vs. Elderly Females, Young Males vs. Elderly Males, Young Females vs. Elderly Males and Young Males vs. Elderly Females.

\begin{tabular}{lccc}
\hline Combinations & Explain variation PC1 $(\%)$ & Explain variation PC2 (\%) & Explain variation total $(\%)$ \\
\hline Elderly Females vs. Young Females & 31.8 & 11.7 & 43.5 \\
Elderly Males vs. Young Males & 31.4 & 12.6 & 44 \\
Elderly Males $v s$. Young Females & 28.6 & 13.2 & 41.8 \\
Elderly Females vs. Young Males & 34.7 & 11.3 & 46 \\
\hline
\end{tabular}


Table S4. The intake of the five macronutrients (protein, fat, sugars, starch and fiber) in 144 elderly subjects, the unit is g.day/BW (BW = body weight).

\begin{tabular}{|c|c|c|c|c|c|}
\hline IDs & Protein.g.day/BW & Fat.g.day/BW & Sugars.g.day/BW & Starch.g.day/BW & Fiber.g.day/BW \\
\hline $0 \_1$ & 1.5557 & 1.7308 & 1.3943 & 1.5925 & 0.5047 \\
\hline $0 \_4$ & 1.5917 & 1.1818 & 1.2859 & 0.7339 & 0.4904 \\
\hline $0 \_6$ & 0.8124 & 0.9441 & 1.1786 & 0.5426 & 0.3100 \\
\hline 0_14 & 1.2005 & 1.4370 & 1.0349 & 0.9942 & 0.3379 \\
\hline 0_15 & 1.1335 & 1.0609 & 0.6636 & 0.5785 & 0.3344 \\
\hline 0_18 & 0.8276 & 1.1431 & 0.7702 & 1.0935 & 0.4259 \\
\hline 0_21 & 1.2249 & 1.4597 & 1.0274 & 0.4520 & 0.2988 \\
\hline $0 \_22$ & 1.1041 & 1.1713 & 0.9903 & 0.8107 & 0.3711 \\
\hline $0 \_23$ & 1.1502 & 0.9825 & 0.8784 & 0.3378 & 0.2394 \\
\hline 0_24 & 1.6821 & 0.6987 & 1.2539 & 0.5713 & 0.3490 \\
\hline $0 \_26$ & 0.4537 & 0.7967 & 0.5523 & 0.7791 & 0.2427 \\
\hline 0_27 & 1.6414 & 1.4389 & 1.1257 & 0.6167 & 0.3200 \\
\hline $0 \_28$ & 0.9324 & 0.8969 & 0.8693 & 0.3251 & 0.3431 \\
\hline 0_29 & 0.9144 & 0.9972 & 0.6749 & 0.9065 & 0.3633 \\
\hline $0 \_31$ & 0.8127 & 1.0097 & 0.9238 & 1.1653 & 0.3557 \\
\hline 0_32 & 0.8526 & 0.6895 & 1.1085 & 0.6441 & 0.2997 \\
\hline $0 \_33$ & 1.0264 & 0.9455 & 0.6264 & 0.3978 & 0.2364 \\
\hline 0_34 & 1.0445 & 1.0670 & 1.0819 & 0.0003 & 0.3771 \\
\hline $0 \_36$ & 0.9269 & 0.7083 & 0.7705 & 0.4615 & 0.2473 \\
\hline $0 \_37$ & 0.6888 & 0.6933 & 0.8141 & 0.1878 & 0.1900 \\
\hline $0 \_38$ & 0.9444 & 0.9177 & 0.4059 & 0.4909 & 0.2464 \\
\hline 0_39 & 1.4399 & 1.7376 & 1.2068 & 0.5891 & 0.3527 \\
\hline 0_40 & 0.8821 & 0.5950 & 0.8962 & 0.4444 & 0.2539 \\
\hline 0_41 & 1.2652 & 0.6951 & 1.1399 & 0.8662 & 0.4452 \\
\hline 0_43 & 2.5328 & 2.0696 & 2.7470 & 2.1055 & 0.7724 \\
\hline 0_44 & 1.0676 & 0.9373 & 1.1071 & 0.4817 & 0.4097 \\
\hline 0_46 & 0.7068 & 0.8154 & 0.9410 & 0.6833 & 0.2592 \\
\hline 0_48 & 1.0110 & 1.1405 & 1.1202 & 0.5473 & 0.3645 \\
\hline 0_49 & 0.9117 & 0.6105 & 0.7132 & 0.7308 & 0.3176 \\
\hline $0 \_51$ & 0.7787 & 0.8696 & 0.1642 & 0.1434 & 0.0819 \\
\hline $0 \_52$ & 1.3128 & 1.1810 & 0.6678 & 0.7520 & 0.3612 \\
\hline $0 \_53$ & 1.2636 & 1.0235 & 0.8009 & 0.9866 & 0.5002 \\
\hline $0 \_55$ & 0.8352 & 0.6172 & 0.2573 & 0.6138 & 0.0951 \\
\hline $0 \_56$ & 1.3581 & 0.8665 & 1.6161 & 0.8574 & 0.3898 \\
\hline $0 \_57$ & 1.5240 & 1.0249 & 1.1763 & 0.1773 & 0.6269 \\
\hline 0_59 & 0.7799 & 0.4208 & 0.1436 & 0.5196 & 0.2487 \\
\hline 0_60 & 0.6462 & 0.4225 & 0.3975 & 0.1021 & 0.1024 \\
\hline
\end{tabular}




\begin{tabular}{|c|c|c|c|c|c|}
\hline 0_61 & 1.0826 & 0.5167 & 0.8088 & 0.3449 & 0.2135 \\
\hline 0_62 & 1.6710 & 1.2644 & 1.8137 & 1.5770 & 0.4442 \\
\hline 0_63 & 1.5554 & 0.9963 & 0.7838 & 1.2630 & 0.4181 \\
\hline 0_64 & 0.9692 & 0.7238 & 0.6139 & 1.2231 & 0.5614 \\
\hline 0_65 & 1.1350 & 1.2568 & 1.0979 & 0.6467 & 0.3782 \\
\hline 0_66 & 1.0871 & 1.0662 & 0.5413 & 0.1512 & 0.1859 \\
\hline 0_67 & 0.7801 & 0.3722 & 0.1907 & 0.8302 & 0.2378 \\
\hline 0_68 & 0.8297 & 0.9113 & 0.4584 & 0.0870 & 0.2222 \\
\hline 0_70 & 1.2450 & 1.1853 & 0.1995 & 0.3491 & 0.1655 \\
\hline 0_73 & 1.3663 & 0.8823 & 0.9784 & 0.4545 & 0.4512 \\
\hline 0_77 & 0.6990 & 0.8736 & 1.9231 & 0.3095 & 0.2975 \\
\hline 0_78 & 0.8593 & 0.7612 & 1.2209 & 0.7574 & 0.2919 \\
\hline 0_79 & 1.0802 & 0.5227 & 1.3970 & 0.2617 & 0.3329 \\
\hline $0 \_80$ & 1.5228 & 0.8298 & 0.5524 & 0.5171 & 0.2282 \\
\hline $0 \_81$ & 1.5409 & 0.7475 & 0.2783 & 0.0677 & 0.2059 \\
\hline $0 \_83$ & 1.3186 & 1.8031 & 0.3946 & 1.7299 & 0.2693 \\
\hline 0_84 & 1.2995 & 1.0488 & 0.8929 & 0.5104 & 0.2005 \\
\hline $0 \_85$ & 1.3312 & 1.1202 & 1.5583 & 0.3174 & 0.2128 \\
\hline 0_88 & 0.9950 & 0.6765 & 0.6779 & 1.1255 & 0.3301 \\
\hline 0_89 & 1.2978 & 1.3769 & 1.6133 & 0.7713 & 0.4382 \\
\hline 0_91 & 1.2345 & 1.3697 & 1.8658 & 0.4805 & 0.3806 \\
\hline 0_94 & 0.9884 & 0.8089 & 0.8910 & 0.5189 & 0.3920 \\
\hline 0_95 & 0.7246 & 0.8106 & 0.5873 & 0.1505 & 0.2546 \\
\hline 0_96 & 0.9055 & 0.6556 & 0.8996 & 0.7494 & 0.3158 \\
\hline 0_97 & 0.7443 & 0.5444 & 0.7418 & 0.5518 & 0.3856 \\
\hline 0_98 & 1.4007 & 0.9556 & 1.3975 & 1.1565 & 0.5376 \\
\hline 0_99 & 1.3554 & 0.8249 & 1.1557 & 1.2471 & 0.5355 \\
\hline 0_100 & 1.3980 & 1.3521 & 0.7047 & 0.9262 & 0.3684 \\
\hline 0_102 & 1.1641 & 1.0940 & 1.3047 & 1.0734 & 0.4606 \\
\hline 0_103 & 0.9412 & 0.4996 & 0.3963 & 0.3139 & 0.2485 \\
\hline 0_104 & 0.8008 & 0.4632 & 0.5266 & 0.2660 & 0.3126 \\
\hline 0_105 & 0.8292 & 0.9298 & 0.3065 & 0.1541 & 0.3234 \\
\hline 0_106 & 0.8156 & 0.5793 & 0.8970 & 0.6946 & 0.3456 \\
\hline 0_108 & 1.1444 & 1.2174 & 1.3625 & 0.6030 & 0.5132 \\
\hline 0_109 & 1.3290 & 2.1761 & 1.4302 & 0.2730 & 0.4246 \\
\hline 0_110 & 1.1701 & 0.7896 & 0.5589 & 0.6653 & 0.3135 \\
\hline 0_111 & 1.3349 & 1.4568 & 0.8620 & 0.3544 & 0.3700 \\
\hline 0_112 & 1.2052 & 1.0271 & 0.5946 & 1.0433 & 0.4703 \\
\hline 0_113 & 1.6064 & 1.5758 & 0.7019 & 0.4018 & 0.5219 \\
\hline 0_115 & 1.3259 & 1.3192 & 0.7218 & 1.7264 & 0.3564 \\
\hline 0_116 & 1.3286 & 0.6668 & 0.4841 & 0.2947 & 0.3009 \\
\hline 0_118 & 1.1079 & 0.8182 & 0.8167 & 0.0148 & 0.3658 \\
\hline 0_120 & 0.8700 & 0.8327 & 0.6136 & 0.2135 & 0.2016 \\
\hline 0_121 & 1.0911 & 1.3955 & 0.6157 & 0.4553 & 0.2862 \\
\hline
\end{tabular}




\begin{tabular}{|c|c|c|c|c|c|}
\hline 0_122 & 1.4766 & 1.0921 & 1.3604 & 0.5223 & 0.5246 \\
\hline 0_123 & 2.2583 & 0.7478 & 1.8325 & 0.2823 & 0.5593 \\
\hline 0_124 & 0.9802 & 1.0667 & 0.7375 & 1.0382 & 0.4054 \\
\hline 0_125 & 1.1725 & 1.0206 & 0.4607 & 0.5439 & 0.2237 \\
\hline 0_128 & 1.0146 & 1.0481 & 1.4533 & 0.5222 & 0.3132 \\
\hline 0_129 & 0.6032 & 0.6742 & 1.0744 & 0.2526 & 0.2571 \\
\hline 0_130 & 1.8276 & 1.7016 & 1.9786 & 1.0777 & 0.5881 \\
\hline 0_131 & 1.0847 & 0.8108 & 0.7439 & 0.3301 & 0.2548 \\
\hline 0_133 & 1.4603 & 1.5218 & 1.3888 & 0.5164 & 0.4500 \\
\hline 0_134 & 1.3940 & 2.0209 & 0.7949 & 0.6626 & 0.3279 \\
\hline 0_135 & 0.7557 & 0.8458 & 1.4703 & 1.2800 & 0.5961 \\
\hline 0_136 & 0.8542 & 0.6699 & 0.8230 & 0.2619 & 0.2032 \\
\hline 0_137 & 1.0650 & 0.8272 & 0.9519 & 0.2859 & 0.3520 \\
\hline 0_138 & 1.2542 & 1.0839 & 2.6769 & 0.4774 & 0.4093 \\
\hline 0_139 & 1.1755 & 1.4887 & 1.0741 & 0.4451 & 0.4494 \\
\hline 0_140 & 1.8588 & 1.2636 & 1.6619 & 0.7626 & 0.5623 \\
\hline 0_142 & 1.0391 & 1.3818 & 0.7648 & 1.3107 & 0.3990 \\
\hline 0_143 & 1.3978 & 1.3378 & 1.5167 & 0.4278 & 0.6837 \\
\hline 0_145 & 0.8197 & 0.5769 & 0.8824 & 0.2859 & 0.2512 \\
\hline 0_146 & 1.6465 & 1.3557 & 1.7180 & 1.0591 & 0.4287 \\
\hline 0_155 & 0.8787 & 0.5963 & 0.3136 & 0.2603 & 0.1262 \\
\hline 0_157 & 0.8288 & 0.8881 & 0.4691 & 0.3905 & 0.2286 \\
\hline 0_159 & 0.8904 & 0.4647 & 1.1241 & 0.4772 & 0.2085 \\
\hline 0_162 & 0.9576 & 1.3797 & 0.4517 & 0.2175 & 0.2618 \\
\hline 0_164 & 1.5601 & 1.1401 & 1.8335 & 1.3283 & 0.5505 \\
\hline 0_165 & 1.2415 & 2.4758 & 1.2862 & 0.9947 & 0.4434 \\
\hline 0_166 & 0.9768 & 0.7717 & 1.0283 & 1.0294 & 0.3239 \\
\hline 0_168 & 1.5382 & 0.7273 & 0.9354 & 1.0196 & 0.2154 \\
\hline 0_169 & 0.9280 & 0.7283 & 1.3410 & 0.3898 & 0.3339 \\
\hline 0_170 & 0.5843 & 0.5381 & 2.1681 & 0.4784 & 0.2116 \\
\hline 0_171 & 1.8174 & 1.3103 & 1.9756 & 0.9559 & 0.6123 \\
\hline 0_172 & 2.2570 & 1.7933 & 2.1235 & 1.5049 & 0.5740 \\
\hline 0_173 & 1.2718 & 0.6837 & 0.6478 & 0.4161 & 0.2473 \\
\hline 0_174 & 0.8639 & 1.4083 & 0.3920 & 0.2234 & 0.0755 \\
\hline 0_175 & 1.4532 & 1.6616 & 1.3260 & 1.0955 & 0.3697 \\
\hline 0_176 & 1.0596 & 0.9619 & 0.6630 & 0.2389 & 0.2651 \\
\hline 0_177 & 0.7607 & 0.7599 & 0.1397 & 0.8710 & 0.1176 \\
\hline 0_179 & 0.7567 & 0.3625 & 0.2783 & 0.1633 & 0.2189 \\
\hline 0_180 & 1.0403 & 0.9130 & 0.4129 & 0.7367 & 0.2706 \\
\hline 0_181 & 0.9403 & 1.2287 & 0.6518 & 0.4028 & 0.2440 \\
\hline 0_182 & 1.2501 & 0.9725 & 0.4542 & 0.2604 & 0.2529 \\
\hline 0_186 & 1.6943 & 0.8272 & 1.2295 & 0.8041 & 0.4360 \\
\hline 0_190 & 0.9570 & 0.7578 & 1.6944 & 0.8263 & 0.3687 \\
\hline 0_192 & 1.1166 & 1.0085 & 0.7883 & 0.3643 & 0.1976 \\
\hline
\end{tabular}




\begin{tabular}{llllll}
$0 \_193$ & 0.5685 & 0.4150 & 0.8455 & 0.4449 & 0.2335 \\
$0 \_194$ & 1.1350 & 1.1131 & 1.2728 & 0.6722 & 0.4243 \\
$0 \_195$ & 1.1152 & 0.9768 & 0.8177 & 0.8379 & 0.2786 \\
$0 \_196$ & 0.9460 & 0.8756 & 1.1901 & 0.6239 & 0.2378 \\
0_199 & 0.9466 & 0.7198 & 0.6082 & 0.6566 & 0.1258 \\
$0 \_202$ & 1.0271 & 0.6334 & 1.1549 & 0.8363 & 0.4500 \\
0_206 & 0.8110 & 0.7924 & 0.7649 & 0.4843 & 0.4667 \\
0_213 & 0.8324 & 0.6838 & 0.5818 & 0.1801 & 0.2673 \\
$0 \_218$ & 1.3540 & 1.8352 & 1.1416 & 1.1349 & 0.2343 \\
$0 \_221$ & 0.6923 & 0.3719 & 0.5668 & 0.2692 & 0.2137 \\
$0 \_224$ & 0.9373 & 1.0617 & 0.7074 & 0.4900 & 0.1973 \\
$0 \_225$ & 1.1078 & 1.0552 & 0.9401 & 0.4175 & 0.2817 \\
$0 \_231$ & 1.3375 & 1.1308 & 0.9135 & 0.5590 & 0.3084 \\
$0 \_232$ & 1.0043 & 1.5106 & 0.6294 & 0.5211 & 0.1859 \\
$0 \_233$ & 1.4848 & 2.0240 & 2.0763 & 1.5959 & 0.4975 \\
$0 \_234$ & 1.3441 & 1.3271 & 1.6220 & 0.6384 & 0.2699 \\
$0 \_236$ & 1.2173 & 0.6799 & 0.7976 & 1.4151 & 0.3242 \\
$0 \_237$ & 0.4676 & 0.4880 & 1.3823 & 0.2664 & 0.2996 \\
$0 \_238$ & 1.5164 & 0.8870 & 0.6057 & 1.8873 & 0.5397 \\
\hline
\end{tabular}


Table S5. ANOVA results on the 36 fecal metabolites comparing High Consumption (HC) versus Low Consumption (LC) for the five selected dietary variables: protein, fat, sugars, starch and fiber intakes. *Significant p-values which have passed Monte Carlo test.

\begin{tabular}{|c|c|c|c|c|c|c|c|c|c|c|c|}
\hline \multirow[b]{2}{*}{ No. } & \multirow[b]{2}{*}{ Name } & \multicolumn{2}{|c|}{ protein } & \multicolumn{2}{|c|}{ fat } & \multicolumn{2}{|c|}{ sugars } & \multirow{2}{*}{$\begin{array}{c}\text { starch } \\
\begin{array}{c}\mathrm{p}- \\
\text { value }\end{array}\end{array}$} & \multicolumn{3}{|c|}{ fiber } \\
\hline & & $\begin{array}{c}\mathrm{p}- \\
\text { value }\end{array}$ & $\begin{array}{c}\text { Effect } \\
\text { size }\end{array}$ & $\begin{array}{c}\mathrm{p}- \\
\text { value }\end{array}$ & $\begin{array}{c}\text { Effect } \\
\text { size }\end{array}$ & $\begin{array}{c}\text { p- } \\
\text { value }\end{array}$ & $\begin{array}{c}\text { Effect } \\
\text { size }\end{array}$ & & $\begin{array}{c}\text { Effect } \\
\text { size }\end{array}$ & $\begin{array}{c}\mathrm{p}- \\
\text { value }\end{array}$ & $\begin{array}{c}\text { Effect } \\
\text { size }\end{array}$ \\
\hline 1 & valeric acid & 0.192 & 1.768 & 0.085 & 3.064 & 0.913 & 0.013 & 0.130 & 2.369 & 0.104 & 2.732 \\
\hline 2 & isovaleric acid & 0.617 & 0.262 & 0.217 & 1.581 & 0.564 & 0.348 & 0.713 & 0.142 & 0.544 & 0.384 \\
\hline 3 & leucine & 0.557 & 0.361 & $0.016^{*}$ & 5.934 & 0.672 & 0.187 & 0.701 & 0.154 & 0.059 & 3.663 \\
\hline 4 & isoleucine & 0.846 & 0.040 & $0.040 *$ & 4.309 & 0.588 & 0.306 & 0.972 & 0.001 & 0.094 & 2.897 \\
\hline 5 & valine & 0.575 & 0.329 & $0.019 *$ & 5.620 & 0.549 & 0.376 & 0.815 & 0.057 & 0.068 & 3.421 \\
\hline 6 & propionic acid & 0.151 & 2.134 & $0.030 *$ & 4.812 & 0.522 & 0.429 & 0.401 & 0.737 & 0.320 & 1.030 \\
\hline 7 & ethanol & 0.599 & 0.288 & 0.890 & 0.020 & 0.900 & 0.017 & 0.777 & 0.084 & 0.665 & 0.196 \\
\hline 8 & lactic acid & 0.526 & 0.420 & 0.302 & 1.109 & 0.308 & 1.083 & 0.227 & 1.519 & 0.325 & 1.010 \\
\hline 9 & alanine & 0.316 & 1.049 & 0.193 & 1.756 & 0.464 & 0.559 & 0.896 & 0.018 & 0.402 & 0.732 \\
\hline 10 & butyric acid & 0.437 & 0.630 & 0.241 & 1.428 & 0.603 & 0.283 & 0.358 & 0.881 & 0.741 & 0.114 \\
\hline 11 & acetic acid & 0.533 & 0.407 & 0.239 & 1.444 & 0.423 & 0.670 & 0.321 & 1.026 & 0.950 & 0.004 \\
\hline 12 & methionine & 0.433 & 0.641 & 0.091 & 2.952 & 0.785 & 0.078 & 0.573 & 0.332 & 0.061 & 3.620 \\
\hline 13 & acetone & 0.662 & 0.200 & 0.924 & 0.009 & 0.389 & 0.773 & 0.406 & 0.720 & 0.530 & 0.413 \\
\hline 14 & glutamic acid & 0.811 & 0.060 & 0.196 & 1.737 & 0.758 & 0.100 & 0.414 & 0.696 & 0.998 & 0.000 \\
\hline 15 & pyruvate & 0.216 & 1.588 & 0.368 & 0.844 & 0.395 & 0.755 & 0.649 & 0.217 & 0.209 & 1.642 \\
\hline 16 & aspartic acid & 0.946 & 0.005 & 0.091 & 2.950 & 0.853 & 0.036 & 0.930 & 0.008 & 0.645 & 0.223 \\
\hline 17 & sarcosine & 0.319 & 1.036 & $0.019 *$ & 5.593 & 0.559 & 0.356 & 0.380 & 0.803 & 0.058 & 3.696 \\
\hline 18 & trimethylamine & 1.000 & $5.75 \mathrm{E}+06$ & 0.299 & $1.12 \mathrm{E}+14$ & 0.168 & $1.97 \mathrm{E}+14$ & 0.609 & 2.7E-01 & 0.599 & 2.9E-01 \\
\hline 19 & lysine & 0.968 & 0.002 & 0.442 & 0.618 & 0.276 & 1.234 & 0.995 & 0.001 & 0.196 & 1.735 \\
\hline 20 & malonic acid & 0.704 & 0.151 & 0.300 & 1.118 & 0.480 & 0.520 & 0.371 & 0.835 & 0.237 & 1.456 \\
\hline 21 & methanol & 0.432 & 0.644 & 0.290 & 1.164 & 0.423 & 0.671 & $0.018^{*}$ & 5.693 & 0.406 & 0.720 \\
\hline 22 & carnitine & 0.437 & 0.631 & 0.291 & 1.161 & 0.395 & 0.755 & 0.418 & 0.686 & 0.454 & 0.585 \\
\hline 23 & glycine & 0.773 & 0.087 & 0.083 & 3.090 & 0.433 & 0.641 & 0.890 & 0.020 & 0.092 & 2.935 \\
\hline 24 & glycerol & 0.828 & 0.050 & 0.275 & 1.239 & 0.485 & 0.510 & 0.870 & 0.028 & 0.579 & 0.321 \\
\hline 25 & guanidinoacetic acid & 0.882 & 0.023 & 0.671 & 0.188 & 0.541 & 0.391 & 0.888 & 0.021 & 0.511 & 0.451 \\
\hline 26 & betaine & 0.891 & 0.020 & 0.129 & 2.379 & 0.655 & 0.209 & 0.751 & 0.106 & 0.667 & 0.194 \\
\hline 27 & threonine & 0.903 & 0.015 & 0.079 & 3.184 & 0.975 & 0.001 & 0.624 & 0.251 & 0.105 & 2.714 \\
\hline 28 & malic acid & 0.720 & 0.135 & 0.787 & 0.076 & 0.588 & 0.307 & 0.247 & 1.392 & 0.107 & 2.679 \\
\hline 29 & glucose & 0.212 & 1.621 & 0.113 & 2.592 & 0.321 & 1.024 & 0.332 & 0.981 & 0.257 & 1.338 \\
\hline 30 & galactose & 0.557 & 0.360 & 0.373 & 0.827 & 0.328 & 0.997 & 0.248 & 1.389 & 0.089 & 2.988 \\
\hline 31 & uracil & 0.774 & 0.087 & 0.297 & 1.132 & 0.396 & 0.752 & 0.604 & 0.281 & 0.115 & 2.569 \\
\hline 32 & fumaric acid & 0.969 & 0.002 & 0.381 & 0.801 & 0.548 & 0.377 & 0.271 & 1.259 & 0.064 & 3.523 \\
\hline 33 & tyrosine & 0.849 & 0.038 & 0.054 & 3.813 & 0.436 & 0.632 & 0.614 & 0.266 & 0.053 & 3.837 \\
\hline 34 & phenylalanine & 0.728 & 0.127 & $0.037 *$ & 4.437 & 0.575 & 0.329 & 0.517 & 0.438 & $0.047 *$ & 4.049 \\
\hline 35 & xanthine & 0.610 & 0.273 & 0.678 & 0.180 & $0.035^{*}$ & 4.556 & 0.345 & 0.928 & 0.768 & 0.091 \\
\hline
\end{tabular}


Table S6. ANOVA results on the 16 fecal metabolites from total 36 metabolites calculated on sex-stratified High Consumption (HC) versus Low Consumption (LC) groups for the selected dietary variables: protein, fat, sugars, starch and fiber (165 elderly subjects were employed in the calculation). The sex- stratified groups included: Case 1= LC male vs. HC male; case 2= LC female vs. HC female; case 3= LC male $v s$. LC female; case 4= LC male $v s$. HC female; case 5= HC male $v s$. HC female; case $6=$ HC male $v s$. HC female. *Significant p-values which have passed Monte Carlo test.

A

\begin{tabular}{|c|c|c|c|c|c|c|c|c|c|c|}
\hline \multicolumn{3}{|c|}{ Information } & $\begin{array}{l}\text { valeric } \\
\text { acid }\end{array}$ & $\begin{array}{l}\text { isovaleric } \\
\text { acid }\end{array}$ & leucine & isoleucine & valine & $\begin{array}{l}\text { propionic } \\
\text { acid }\end{array}$ & sarcosine & trimethylamine \\
\hline \multirow{12}{*}{ Protein } & \multirow{2}{*}{$\begin{array}{c}\text { LCM vs. } \\
\text { HCM }\end{array}$} & $\begin{array}{c}\mathrm{p}- \\
\text { value }\end{array}$ & 0.510 & 0.872 & 0.590 & 0.700 & 0.554 & 0.952 & 0.368 & 0.595 \\
\hline & & $\begin{array}{c}\text { Effect } \\
\text { size }\end{array}$ & 0.931 & 0.056 & 0.622 & 0.319 & 0.750 & 0.008 & 1.727 & 0.606 \\
\hline & \multirow{2}{*}{$\begin{array}{c}\text { LCF vs. } \\
\text { HCF }\end{array}$} & $\begin{array}{c}\text { p- } \\
\text { value }\end{array}$ & 0.153 & 0.215 & 0.777 & 0.661 & 0.700 & 0.027 & 0.623 & 0.472 \\
\hline & & $\begin{array}{l}\text { Effect } \\
\text { size }\end{array}$ & 4.487 & 3.400 & 0.181 & 0.432 & 0.334 & 10.455 & 0.542 & 1.156 \\
\hline & \multirow{2}{*}{$\begin{array}{c}\text { LCM } v s . \\
\text { LCF }\end{array}$} & $\begin{array}{c}\mathrm{p}- \\
\text { value }\end{array}$ & 0.604 & 0.424 & 0.886 & 0.836 & 0.927 & 0.668 & 0.781 & 0.320 \\
\hline & & $\begin{array}{c}\text { Effect } \\
\text { size }\end{array}$ & 0.604 & 1.426 & 0.046 & 0.097 & 0.019 & 0.412 & 0.174 & 2.198 \\
\hline & \multirow{2}{*}{$\begin{array}{c}\text { LCM vs. } \\
\text { HCF }\end{array}$} & $\begin{array}{c}\mathrm{p}- \\
\text { value }\end{array}$ & 0.060 & 0.038 & 0.873 & 0.519 & 0.753 & 0.039 & 0.872 & 0.625 \\
\hline & & $\begin{array}{l}\text { Effect } \\
\text { size }\end{array}$ & 7.477 & 9.012 & 0.056 & 0.909 & 0.217 & 8.947 & 0.057 & 0.525 \\
\hline & \multirow{2}{*}{$\underset{\text { LCM } v s .}{\text { LCF }}$} & $\begin{array}{c}\mathrm{p}- \\
\text { value }\end{array}$ & 0.883 & 0.372 & 0.697 & 0.580 & 0.619 & 0.710 & 0.154 & 0.601 \\
\hline & & $\begin{array}{l}\text { Effect } \\
\text { size }\end{array}$ & 0.048 & 1.738 & 0.333 & 0.669 & 0.542 & 0.302 & 4.365 & 0.600 \\
\hline & \multirow{2}{*}{$\underset{\text { HCM } v s .}{\text { HCF }}$} & $\begin{array}{c}\mathrm{p}- \\
\text { value }\end{array}$ & 0.203 & 0.041 & 0.521 & 0.352 & 0.417 & 0.037 & 0.423 & 0.898 \\
\hline & & $\begin{array}{c}\text { Effect } \\
\text { size }\end{array}$ & 3.426 & 8.622 & 0.881 & 1.84 & 1.408 & 8.935 & 1.371 & 0.035 \\
\hline \multirow{11}{*}{ Fat } & \multirow{2}{*}{$\begin{array}{c}\text { LCM vs. } \\
\text { HCM }\end{array}$} & $\begin{array}{c}\mathrm{p}- \\
\text { value }\end{array}$ & 0.221 & 0.306 & 0.544 & 0.660 & 0.493 & 0.557 & 0.048 & 0.123 \\
\hline & & $\begin{array}{c}\text { Effect } \\
\text { size }\end{array}$ & 3.170 & 2.228 & 0.789 & 0.414 & 1.006 & 0.737 & 8.031 & 4.974 \\
\hline & \multirow{2}{*}{$\begin{array}{c}\text { LCF vs. } \\
\text { HCF }\end{array}$} & $\begin{array}{c}\mathrm{p}- \\
\text { value }\end{array}$ & 0.110 & 0.308 & 0.020 & 0.048 & 0.032 & 0.007 & 0.318 & 0.893 \\
\hline & & $\begin{array}{c}\text { Effect } \\
\text { size }\end{array}$ & 5.586 & 2.307 & 11.498 & 8.394 & 9.781 & 15.233 & 2.218 & 0.041 \\
\hline & \multirow{2}{*}{$\begin{array}{l}\text { LCM vs. } \\
\text { LCF }\end{array}$} & $\begin{array}{c}\mathrm{p}- \\
\text { value }\end{array}$ & 0.150 & 0.039 & 0.659 & 0.426 & 0.651 & 0.787 & 0.425 & 0.813 \\
\hline & & $\begin{array}{l}\text { Effect } \\
\text { size }\end{array}$ & 4.559 & 9.098 & 0.437 & 1.417 & 0.459 & 0.164 & - & 0.125 \\
\hline & \multirow{2}{*}{$\begin{array}{c}\text { LCM vs. } \\
\text { HCF }\end{array}$} & $\begin{array}{c}\mathrm{p}- \\
\text { value }\end{array}$ & 0.003 & 0.002 & 0.048 & 0.177 & 0.072 & 0.005 & 0.092 & 0.908 \\
\hline & & $\begin{array}{c}\text { Effect } \\
\text { size }\end{array}$ & 17.428 & 18.397 & 8.224 & 3.919 & 6.851 & 15.703 & 6.059 & 0.029 \\
\hline & \multirow{2}{*}{$\underset{\text { LCM } v s .}{\text { LCF }}$} & $\begin{array}{c}\mathrm{p}- \\
\text { value }\end{array}$ & 0.771 & 0.309 & 0.308 & 0.242 & 0.276 & 0.421 & 0.205 & 0.059 \\
\hline & & $\begin{array}{l}\text { Effect } \\
\text { size }\end{array}$ & 0.185 & 2.251 & 2.261 & 2.962 & 2.569 & 1.413 & 3.471 & 7.507 \\
\hline & $\begin{array}{c}\text { HCM vs. } \\
\text { HCF }\end{array}$ & $\begin{array}{c}\mathrm{p}- \\
\text { value }\end{array}$ & 0.051 & 0.047 & 0.172 & 0.370 & 0.279 & 0.011 & 0.888 & 0.067 \\
\hline
\end{tabular}




\begin{tabular}{|c|c|c|c|c|c|c|c|c|c|c|}
\hline & & $\begin{array}{c}\text { Effect } \\
\text { size }\end{array}$ & 7.861 & 8.125 & 3.935 & 1.716 & 2.489 & 13.039 & 0.043 & 6.982 \\
\hline \multirow{12}{*}{ Sugars } & \multirow{2}{*}{$\begin{array}{c}\text { LCM vs. } \\
\text { HCM }\end{array}$} & $\begin{array}{c}\mathrm{p}- \\
\text { value }\end{array}$ & 0.338 & 0.256 & 0.499 & 0.442 & 0.505 & 0.063 & 0.106 & 0.037 \\
\hline & & $\begin{array}{c}\text { Effect } \\
\text { size }\end{array}$ & 1.952 & 2.739 & 0.979 & 1.264 & 0.953 & 7.152 & 5.472 & 8.911 \\
\hline & \multirow{2}{*}{$\begin{array}{c}\text { LCF vs. } \\
\text { HCF }\end{array}$} & $\begin{array}{c}\mathrm{p}- \\
\text { value }\end{array}$ & 0.539 & 0.605 & 0.620 & 0.447 & 0.461 & 0.550 & 0.800 & 0.907 \\
\hline & & $\begin{array}{c}\text { Effect } \\
\text { size }\end{array}$ & 0.846 & 0.598 & 0.550 & 1.292 & 1.212 & 0.800 & 0.144 & 0.031 \\
\hline & \multirow{2}{*}{$\begin{array}{c}\text { LCM vs. } \\
\text { LCF }\end{array}$} & $\begin{array}{c}\mathrm{p}- \\
\text { value }\end{array}$ & 0.950 & 0.441 & 0.468 & 0.595 & 0.467 & 0.571 & 0.247 & 0.384 \\
\hline & & $\begin{array}{c}\text { Effect } \\
\text { size }\end{array}$ & 0.009 & 1.327 & 1.176 & 0.634 & 1.181 & 0.719 & 2.967 & 1.691 \\
\hline & \multirow{2}{*}{$\begin{array}{c}\text { LCM vs. } \\
\text { HCF }\end{array}$} & $\begin{array}{c}\mathrm{p}- \\
\text { value }\end{array}$ & 0.509 & 0.201 & 0.847 & 0.775 & 0.968 & 0.922 & 0.392 & 0.394 \\
\hline & & $\begin{array}{c}\text { Effect } \\
\text { size }\end{array}$ & 0.954 & 3.534 & 0.082 & 0.180 & 0.003 & 0.021 & 1.597 & 1.582 \\
\hline & \multirow{2}{*}{$\begin{array}{c}\text { HCM vs. } \\
\text { LCF }\end{array}$} & $\begin{array}{c}\mathrm{P}- \\
\text { value }\end{array}$ & 0.296 & 0.066 & 0.925 & 0.887 & 0.902 & 0.301 & 0.613 & 0.264 \\
\hline & & $\begin{array}{c}\text { Effect } \\
\text { size }\end{array}$ & 2.367 & 7.164 & 0.020 & 0.044 & 0.033 & 2.325 & 0.560 & 2.707 \\
\hline & \multirow{2}{*}{$\begin{array}{c}\text { HCM } v s . \\
\text { HCF }\end{array}$} & $\begin{array}{c}\mathrm{p}- \\
\text { value }\end{array}$ & 0.099 & 0.021 & 0.663 & 0.315 & 0.496 & 0.078 & 0.487 & 0.145 \\
\hline & & $\begin{array}{c}\text { Effect } \\
\text { size }\end{array}$ & 5.697 & 10.822 & 0.408 & 2.146 & 0.990 & 6.476 & 1.034 & 4.461 \\
\hline \multirow{12}{*}{ Starch } & \multirow{2}{*}{$\begin{array}{c}\text { LCM vs. } \\
\text { HCM }\end{array}$} & $\begin{array}{c}\mathrm{p}- \\
\text { value }\end{array}$ & 0.496 & 0.841 & 0.202 & 0.282 & 0.202 & 0.571 & 0.974 & 0.873 \\
\hline & & $\begin{array}{c}\text { Effect } \\
\text { size }\end{array}$ & 0.992 & 0.086 & 3.435 & 2.461 & 3.445 & 0.687 & 0.002 & 0.055 \\
\hline & \multirow{2}{*}{$\begin{array}{c}\text { LCF vs. } \\
\text { HCF }\end{array}$} & $\begin{array}{c}\mathrm{p}- \\
\text { value }\end{array}$ & 0.073 & 0.248 & 0.961 & 0.784 & 0.776 & 0.303 & 0.388 & 0.331 \\
\hline & & $\begin{array}{c}\text { Effect } \\
\text { size }\end{array}$ & 6.975 & 2.955 & 0.005 & 0.169 & 0.181 & 2.358 & 1.659 & 2.104 \\
\hline & \multirow{2}{*}{$\begin{array}{l}\text { LCM vs. } \\
\text { LCF }\end{array}$} & $\begin{array}{c}\mathrm{p}- \\
\text { value }\end{array}$ & 0.283 & 0.224 & 0.240 & 0.429 & 0.277 & 0.431 & 0.381 & 0.745 \\
\hline & & $\begin{array}{c}\text { Effect } \\
\text { size }\end{array}$ & 2.552 & 3.268 & 3.059 & 1.395 & 2.618 & 1.382 & 1.713 & 0.238 \\
\hline & \multirow{2}{*}{$\begin{array}{l}\text { LCM vs. } \\
\text { HCF }\end{array}$} & $\begin{array}{c}\mathrm{p}- \\
\text { value }\end{array}$ & 0.007 & 0.025 & 0.320 & 0.664 & 0.466 & 0.079 & 0.966 & 0.549 \\
\hline & & $\begin{array}{c}\text { Effect } \\
\text { size }\end{array}$ & 14.954 & 10.487 & 2.153 & 0.414 & 1.159 & 6.540 & 0.004 & 0.787 \\
\hline & \multirow{2}{*}{$\begin{array}{l}\text { HCM } v s . \\
\text { LCF }\end{array}$} & $\begin{array}{c}\mathrm{p}- \\
\text { value }\end{array}$ & 0.655 & 0.153 & 0.852 & 0.747 & 0.806 & 0.822 & 0.495 & 0.892 \\
\hline & & $\begin{array}{c}\text { Effect } \\
\text { size }\end{array}$ & 0.437 & 4.382 & 0.077 & 0.229 & 0.132 & 0.111 & 1.016 & 0.041 \\
\hline & \multirow{2}{*}{$\begin{array}{c}\text { HCM } v s . \\
\text { HCF }\end{array}$} & $\begin{array}{c}\mathrm{p}- \\
\text { value }\end{array}$ & 0.024 & 0.014 & 0.834 & 0.585 & 0.619 & 0.212 & 0.999 & 0.468 \\
\hline & & $\begin{array}{l}\text { Effect } \\
\text { size }\end{array}$ & 10.413 & 12.146 & 0.095 & 0.640 & 0.530 & 3.295 & - & 1.125 \\
\hline \multirow{9}{*}{ Fiber } & \multirow{2}{*}{$\begin{array}{c}\text { LCM vs. } \\
\text { HCM }\end{array}$} & $\begin{array}{c}\mathrm{p}- \\
\text { value }\end{array}$ & 0.341 & 0.789 & 0.128 & 0.072 & 0.108 & 0.917 & 0.084 & 0.074 \\
\hline & & $\begin{array}{c}\text { Effect } \\
\text { size }\end{array}$ & 1.928 & 0.154 & 4.862 & 6.707 & 5.392 & 0.023 & 6.234 & 6.637 \\
\hline & \multirow{2}{*}{$\begin{array}{c}\text { LCF vs. } \\
\text { HCF }\end{array}$} & $\begin{array}{c}\mathrm{p}- \\
\text { value }\end{array}$ & 0.111 & 0.413 & 0.735 & 0.921 & 0.887 & 0.212 & 0.611 & 0.718 \\
\hline & & $\begin{array}{c}\text { Effect } \\
\text { size }\end{array}$ & 5.539 & 1.494 & 0.257 & 0.022 & 0.046 & 3.447 & 0.580 & 0.292 \\
\hline & \multirow{2}{*}{$\begin{array}{l}\text { LCM vs. } \\
\text { LCF }\end{array}$} & $\begin{array}{c}\mathrm{p}- \\
\text { value }\end{array}$ & 0.567 & 0.316 & 0.692 & 0.716 & 0.708 & 0.832 & 0.653 & 0.718 \\
\hline & & $\begin{array}{c}\text { Effect } \\
\text { size }\end{array}$ & 0.735 & 2.230 & 0.351 & 0.297 & 0.314 & 0.101 & 0.452 & 0.294 \\
\hline & \multirow{2}{*}{$\begin{array}{c}\text { LCM vs. } \\
\text { HCF }\end{array}$} & $\begin{array}{c}\mathrm{p}- \\
\text { value }\end{array}$ & 0.054 & 0.078 & 0.458 & 0.821 & 0.611 & 0.243 & 0.373 & 0.988 \\
\hline & & $\begin{array}{c}\text { Effect } \\
\text { size }\end{array}$ & 7.818 & 6.590 & 1.201 & 0.113 & 0.566 & 2.946 & 1.732 & 0.000 \\
\hline & $\begin{array}{c}\text { HCM vs. } \\
\text { LCF }\end{array}$ & $\begin{array}{c}\mathrm{p}- \\
\text { value }\end{array}$ & 0.646 & 0.498 & 0.290 & 0.121 & 0.229 & 0.759 & 0.142 & 0.163 \\
\hline
\end{tabular}




\begin{tabular}{|c|c|l|l|l|l|l|l|l|l|} 
& $\begin{array}{c}\text { Effect } \\
\text { size }\end{array}$ & 0.461 & 1.003 & 2.434 & 5.153 & 3.130 & 0.207 & 4.621 & 4.192 \\
\hline \multirow{2}{*}{$\begin{array}{c}\text { HCM vs. } \\
\text { HCF }\end{array}$} & $\begin{array}{c}\mathrm{p}- \\
\text { value }\end{array}$ & 0.296 & 0.163 & 0.495 & 0.153 & 0.308 & 0.282 & 0.381 & 0.063 \\
\cline { 2 - 9 } & $\begin{array}{c}\text { Effect } \\
\text { size }\end{array}$ & 2.318 & 4.097 & 0.997 & 4.296 & 2.211 & 2.454 & 1.640 & 7.181 \\
\hline
\end{tabular}

B

\begin{tabular}{|c|c|c|c|c|c|c|c|c|c|c|}
\hline \multicolumn{3}{|c|}{ Information } & $\begin{array}{l}\text { malonic } \\
\text { acid }\end{array}$ & methanol & threonine & $\begin{array}{l}\text { malic } \\
\text { acid }\end{array}$ & $\begin{array}{l}\text { fumaric } \\
\text { acid }\end{array}$ & tyrosine & phenylalanine & xanthine \\
\hline \multirow{12}{*}{ Protein } & \multirow{2}{*}{$\begin{array}{l}0.302 \\
2.267\end{array}$} & 0.948 & 0.891 & 0.576 & 0.285 & 0.923 & 0.825 & 0.665 & 0.368 & 0.595 \\
\hline & & 0.009 & 0.040 & 0.669 & 2.431 & 0.020 & 0.105 & 0.403 & 1.727 & 0.606 \\
\hline & \multirow{2}{*}{$\begin{array}{l}0.474 \\
1.143\end{array}$} & 0.672 & 0.974 & 0.262 & 0.326 & 0.547 & 0.628 & 0.388 & 0.623 & 0.472 \\
\hline & & 0.403 & 0.002 & 2.788 & 2.141 & 0.812 & 0.525 & 1.660 & 0.542 & 1.156 \\
\hline & \multirow{2}{*}{$\begin{array}{l}0.219 \\
3.341\end{array}$} & 0.435 & 0.360 & 0.414 & 0.121 & 0.570 & 0.774 & 0.947 & 0.781 & 0.320 \\
\hline & & 1.362 & 1.866 & 1.491 & 5.263 & 0.722 & 0.185 & 0.010 & 0.174 & 2.198 \\
\hline & \multirow{2}{*}{$\begin{array}{l}0.364 \\
1.792\end{array}$} & 0.721 & 0.466 & 0.789 & 0.573 & 0.215 & 0.431 & 0.287 & 0.872 & 0.625 \\
\hline & & 0.280 & 1.161 & 0.157 & 0.694 & 3.318 & 1.356 & 2.464 & 0.057 & 0.525 \\
\hline & \multirow{2}{*}{$\begin{array}{l}0.419 \\
1.428\end{array}$} & 0.540 & 0.489 & 0.808 & 0.722 & 0.586 & 0.636 & 0.741 & 0.154 & 0.601 \\
\hline & & 0.820 & 1.044 & 0.130 & 0.278 & 0.650 & 0.490 & 0.240 & 4.365 & 0.600 \\
\hline & \multirow{2}{*}{$\begin{array}{l}0.931 \\
0.016\end{array}$} & 0.807 & 0.570 & 0.400 & 0.571 & 0.263 & 0.348 & 0.633 & 0.423 & 0.898 \\
\hline & & 0.129 & 0.691 & 1.511 & 0.689 & 2.662 & 1.879 & 0.488 & 1.371 & 0.035 \\
\hline \multirow{12}{*}{ Fat } & \multirow{2}{*}{$\begin{array}{l}0.476 \\
1.087\end{array}$} & 0.864 & 0.852 & 0.118 & 0.252 & 0.846 & 0.958 & 0.195 & 0.048 & 0.123 \\
\hline & & 0.063 & 0.075 & 5.128 & 2.781 & 0.081 & 0.006 & 3.549 & 8.031 & 4.974 \\
\hline & \multirow{2}{*}{$\begin{array}{l}0.177 \\
4.014\end{array}$} & 0.562 & 0.061 & 0.043 & 0.029 & 0.023 & 0.017 & 0.715 & 0.318 & 0.893 \\
\hline & & 0.755 & 7.587 & 8.829 & 10.119 & 10.991 & 11.947 & 0.299 & 2.218 & 0.041 \\
\hline & \multirow{2}{*}{$\begin{array}{l}0.178 \\
4.002\end{array}$} & 0.207 & 0.289 & 0.243 & 0.133 & 0.051 & 0.106 & 0.046 & 0.425 & 0.813 \\
\hline & & 3.510 & 2.496 & 3.018 & 4.934 & 8.169 & 5.705 & 8.560 & - & 0.125 \\
\hline & \multirow{2}{*}{$\begin{array}{l}0.994 \\
0.000\end{array}$} & 0.583 & 0.310 & 0.330 & 0.225 & 0.524 & 0.279 & 0.124 & 0.092 & 0.908 \\
\hline & & 0.661 & 2.243 & 2.062 & 3.190 & 0.890 & 2.546 & 5.069 & 6.059 & 0.029 \\
\hline & \multirow{2}{*}{$\begin{array}{l}0.300 \\
2.331\end{array}$} & 0.187 & 0.244 & 0.740 & 0.678 & 0.114 & 0.158 & 0.459 & 0.205 & 0.059 \\
\hline & & 3.757 & 2.934 & 0.241 & 0.377 & 5.332 & 4.280 & 1.198 & 3.471 & 7.507 \\
\hline & \multirow{2}{*}{$\begin{array}{l}0.471 \\
1.110\end{array}$} & 0.510 & 0.423 & 0.014 & 0.055 & 0.454 & 0.306 & 0.738 & 0.888 & 0.067 \\
\hline & & 0.929 & 1.370 & 12.108 & 7.608 & 1.197 & 2.231 & 0.241 & 0.043 & 6.982 \\
\hline \multirow{3}{*}{ Sugars } & \multirow{2}{*}{$\begin{array}{l}0.684 \\
0.355\end{array}$} & 0.223 & 0.223 & 0.677 & 0.638 & 0.267 & 0.459 & 0.449 & 0.106 & 0.037 \\
\hline & & 3.140 & 3.141 & 0.372 & 0.475 & 2.618 & 1.173 & 1.223 & 5.472 & 8.911 \\
\hline & 0.777 & 0.597 & 0.736 & 0.722 & 0.098 & 0.245 & 0.485 & 0.014 & 0.800 & 0.907 \\
\hline
\end{tabular}




\begin{tabular}{|c|c|c|c|c|c|c|c|c|c|c|}
\hline & 0.180 & 0.626 & 0.255 & 0.285 & 5.960 & 2.992 & 1.091 & 12.634 & 0.144 & 0.031 \\
\hline & 0.342 & 0.040 & 0.580 & 0.868 & 0.245 & 0.593 & 0.706 & 0.755 & 0.247 & 0.384 \\
\hline & 2.012 & 9.063 & 0.687 & 0.062 & 2.991 & 0.639 & 0.320 & 0.219 & 2.967 & 1.691 \\
\hline & & 0.120 & 0.887 & 0.592 & 0.549 & 0.523 & 0.721 & 0.033 & 0.392 & 0.394 \\
\hline & 3.351 & 5.162 & 0.045 & 0.630 & 0.788 & 0.891 & 0.280 & 9.489 & 1.597 & 1.582 \\
\hline & 0.445 & 0.517 & 0.573 & 0.805 & 0.519 & 0.575 & 0.763 & 0.301 & 0.613 & 0.264 \\
\hline & 1.273 & 0.921 & 0.695 & 0.134 & 0.908 & 0.687 & 0.200 & 2.328 & 0.560 & 2.707 \\
\hline & 0.429 & 0.868 & 0.387 & 0.928 & 0.339 & 0.079 & 0.283 & 0.232 & 0.487 & 0.145 \\
\hline & 1.336 & 0.059 & 1.598 & 0.018 & 1.944 & 6.409 & 2.447 & 3.030 & 1.034 & 4.461 \\
\hline \multirow{12}{*}{ Starch } & \multirow{2}{*}{$\begin{array}{l}0.285 \\
2.428\end{array}$} & 0.046 & 0.036 & 0.256 & 0.263 & 0.088 & 0.133 & 0.673 & 0.974 & 0.873 \\
\hline & & 8.206 & 9.033 & 2.735 & 2.657 & 6.057 & 4.738 & 0.383 & 0.002 & 0.055 \\
\hline & \multirow{2}{*}{$\begin{array}{l}0.694 \\
0.347\end{array}$} & 0.116 & 0.720 & 0.431 & 0.266 & 0.818 & 0.898 & 0.355 & 0.388 & 0.331 \\
\hline & & 5.394 & 0.288 & 1.385 & 2.737 & 0.119 & 0.037 & 1.903 & 1.659 & 2.104 \\
\hline & \multirow{2}{*}{$\begin{array}{l}0.780 \\
0.175\end{array}$} & 0.591 & 0.237 & 0.717 & 0.842 & 0.480 & 0.399 & 0.832 & 0.381 & 0.745 \\
\hline & & 0.649 & 3.098 & 0.295 & 0.090 & 1.117 & 1.585 & 0.102 & 1.713 & 0.238 \\
\hline & \multirow{2}{*}{$\begin{array}{l}0.604 \\
0.590\end{array}$} & 0.354 & 0.514 & 0.258 & 0.362 & 0.675 & 0.377 & 0.433 & 0.966 & 0.549 \\
\hline & & 1.871 & 0.932 & 2.772 & 1.813 & 0.386 & 1.704 & 1.341 & 0.004 & 0.787 \\
\hline & \multirow{2}{*}{$\begin{array}{l}0.148 \\
4.500\end{array}$} & 0.007 & 0.312 & 0.427 & 0.195 & 0.241 & 0.469 & 0.559 & 0.495 & 0.892 \\
\hline & & 14.813 & 2.219 & 1.380 & 3.631 & 2.981 & 1.145 & 0.747 & 1.016 & 0.041 \\
\hline & \multirow{2}{*}{$\begin{array}{l}0.343 \\
1.916\end{array}$} & 0.203 & 0.255 & 0.975 & 0.729 & 0.225 & 0.612 & 0.777 & 0.999 & 0.468 \\
\hline & & 3.424 & 2.751 & 0.002 & 0.258 & 3.112 & 0.551 & 0.172 & - & 1.125 \\
\hline \multirow{12}{*}{ Fiber } & \multirow{2}{*}{$\begin{array}{l}0.007 \\
14.694\end{array}$} & 0.479 & 0.044 & 0.651 & 0.960 & 0.121 & 0.166 & 0.289 & 0.084 & 0.074 \\
\hline & & 1.072 & 8.350 & 0.439 & 0.005 & 5.033 & 4.049 & 2.391 & 6.234 & 6.637 \\
\hline & & 0.356 & 0.768 & 0.087 & 0.028 & 0.725 & 0.561 & 0.383 & 0.611 & 0.718 \\
\hline & 0.523 & 1.895 & 0.195 & 6.363 & 10.337 & 0.278 & 0.756 & 1.695 & 0.580 & 0.292 \\
\hline & & 0.013 & 0.377 & 0.769 & 0.178 & 0.856 & 0.839 & 0.122 & 0.653 & 0.718 \\
\hline & 0.010 & 12.957 & 1.739 & 0.194 & 4.001 & 0.074 & 0.093 & 5.229 & 0.452 & 0.294 \\
\hline & & 0.102 & 0.626 & 0.188 & 0.207 & 0.861 & 0.677 & 0.496 & 0.373 & 0.988 \\
\hline & 0.638 & 5.703 & 0.520 & 3.745 & 3.435 & 0.067 & 0.381 & 1.013 & 1.732 & 0.000 \\
\hline & & 0.080 & 0.223 & 0.428 & 0.200 & 0.078 & 0.136 & 0.623 & 0.142 & 0.163 \\
\hline & 11.098 & 6.521 & 3.217 & 1.369 & 3.548 & 6.581 & 4.778 & 0.529 & 4.621 & 4.192 \\
\hline & & 0.379 & 0.197 & 0.329 & 0.232 & 0.188 & 0.385 & 0.699 & 0.381 & 0.063 \\
\hline & 5.106 & 1.648 & 3.514 & 2.030 & 3.023 & 3.663 & 1.612 & 0.320 & 1.640 & 7.181 \\
\hline
\end{tabular}

